\title{
Society for Social Medicine
}

The Society for Social Medicine held its annual scientific meeting at Royal Holloway, University of London from 13-15 September 1994. The abstracts of oral presentations are published below.

PLENARY PRESENTATIONS

Ethnicity, race, health, and research: black box, junk, or enlightened epidemiology?

R S BHOPAL (Department of Epidemiology and Public Health, University of Newcastle upon Tyne)

Introduction - The description of human characteristics, including health, by racial group has beguiled researchers in many disciplines, including epidemiology. Some careers have been ruined - in hindsight by racist, prejudiced, or simply misguided work - in this highly complex and controversial arena of research. With the concept of race under attack, ethnicity has now occupied the vacated ground. Ethnicity has become a key, almost routine, epidemiological variable and there is strong pressure on researchers to collect information on it. There have been repeated and enthusiastic claims, by researchers, policy makers, and health planners, about the value of ethnicity and health research. This paper presents a critical analysis of the past and potential value of ethnicity, race, and health research based on epidemiological methods. The key questions are whether such research can inform our understanding of disease causation or help develop appropriate health services (enlightenment), add to our collection of unexplained associations (blackbox epidemiology), or is merely information of little value (junk).

Approach to the paper - A historical analysis will subject the claims of race and health researchers in the past to the reality of today. In addition, hypotheses will be extracted from the epidemiological literature of the 1970s and 1980s. The number of hypotheses which have been pursued and either rejected or supported by subsequent work will be the critical factor in assessing the value of ethnicity and health research in understanding disease causation. Furthermore, the quality of the research that has been done and is underway will be explored in relation to the definition of terms, validity of measurement of ethnicity, quality control of data collection, and the statement of hypotheses which permits their testing. The extent to which studies done in the late 1970s and early 1980s have been used to create appropriate health policy and health plans will be reviewed.

Results of the analysis to date - The work for this paper is not complete but the preliminary findings are that claims of race, ethnicity, and health researchers are highly exaggerated and that information from these studies is hard to utilise for aetiological or service applications. There are grave problems with the way researchers have collected and presented information on ethnicity and health, and with the validity of data collection used in cross cultural studies. The health services have found it difficult to respond to meet the needs of ethnic minority groups and it is unclear whether research has helped or hindered.

From a historical point of view it will be argued that race research has done more harm than good, with many studies breaching research ethics and principles. It is highly pertinent to examine whether ethnicity and health research may also be breaching such principles. This analysis will argue for deeper analysis of the value of ethnicity and health research in epidemiology so that its strengths and limitations can be more widely understood; exaggerated claims are not made; and expectations of researchers, policy makers, and ethnic minorities are maintained at realistic levels.

Gender differences in health: are things really as simple as they seem?

SALLY MACINTYRE, KATE HUNT, HELEN SWEETING (MRC Medical Sociology Unit, Glasgow) Objective - To examine gender differences in self reported health at different points in the life course in two recent British studies.

Design - The prevalence of poorer health reported by men and women to nurse-interviewers was examined in two comparable studies - one a national sample of adults (health and lifestyle survey), the other a regionally based longitudinal study of men and women in early adulthood, and early and late mid-life (west of Scotland twenty07 study).

Setting - National sample, and west of Scotland.

Subjects - Altogether 9000 men and women aged 18 and over living in Britain, focussing particularly on those aged $18-22(n=809)$, 36-40 $(n=971)$, and $56-60$ years $(n=679)$ and 90818 year olds, 85239 year olds, and 85858 year olds living in the central Clydeside conurbation.

Main outcome measures - Three general measures of self assessed health (self assessed health for age, presence and type of long standing illness, and presence of limiting long standing illness) and three of current symptomatolo $y$ (total number of symptoms, number of malaise symptoms, and number of physical symptoms in last month).

Results - The findings from the two studies are consistent. There is no significant difference in the prevalence of long standing illness or limiting long standing illness at any of the three stages of the life cycle examined in either study or in self assessed health except among 18 year olds in the west of Scotland. Female excesses are consistently seen for the mean number of all symptoms and malaise symptoms, though not for physical symptoms. When the symptoms and chronic conditions reported are examined in more detail, however, only a minority show a consistent female excess at each of the life cycle stages, and several show no sex differences at any age in either study.

Conclusion - Detailed inspection of recen evidence from two recent British data sets challenges the conventional wisdom in medical sociology and social epidemiology that women, despite their mortality advantage, have poorer health than men. The direction and magnitude of sex differences in health vary according to the particular measures of health used and according to the phase of the life cycle.

The effects of screening on breast cancer incidence and mortality

M J QUINN, E J ALLEN (Population and Health Statistics, Office of Population Censuses and Surveys, London; on behalf of the United Kingdom Association of Cancer Registries)

Objective - To assess the effects of the NHS breast screening programme on breast cancer incidence and mortality.

Design - Comparison of age specific incidence and mortality before and after the introduction of screening in the late 1980s.

Setting - England and Wales.

Subjects - Women aged over 30 years.

Results - In 1992, the age standardised incidence of breast cancer was $40 \%$ higher than in 1979. After the introduction of screening in 1988, recorded incidence rates rose in the screened age group but not in others. In 1992, the rates levelled off, at values some $25 \%$ higher than in 1987. The age distribution of cases in 1992 compared with that for 1987 clearly shows increases in the screened age groups and decreases in the more elderly. Total mortality from breast cancer has increased since the $1950 \mathrm{~s}$, and rates increased earlier in the younger age groups. By the mid$1980 \mathrm{~s}$, rates had begun to fall in the younger age groups but total mortality was among the highest in the world. Age standardised mortality in the 55-69 age group changed little during the first three years of screening but subsequently fell steeply. When the effects of several changes to the coding of death certificates in 1993 are taken into account, mortality was about $10 \%$ lower than in 1987 . Conclusions - Since the introduction of screening there have been changes in breast cancer incidence and mortality in the screened age group - pronounced increases in incidence (with corresponding decreases among older women) and a steep decrease in mortality which began three years after screening started. The reduction in mortality cannot have resulted from the screening, but if the current trend continues, the Health of the Nation target of a $25 \%$ reduction by the year 2000 will be achieved.

Trends in referral and prescribing patterns in fundholding and non-fundholding practices in the Oxford region, 1990-94

ANGELA COULTER, JEAN BRADLOW, REBECCA SURENDER, SARAH STEWART-BROWN, HELEN DOLL. (Health Services Research Unit, Department of Public Health and Primary Care, University of Oxford)

Introduction - General practice fundholding, now in its fifth year of operation, continues to fuel controversy and there is little consensus about the relative merits of the scheme. The 
Oxford region fundholding study is one of a very small number of studies designed to measure the effect on referral and prescribing decisions when general practitioners have to pay for their patients' prescriptions and consultations with specialists. Interim findings after one year of fundholding have been published. This paper presents follow up results three years after the introduction of the scheme.

Objective - To compare outpatient referral patterns and prescribing rates and costs in fundholding and non-fundholding practices before and after the implementation of the NHS reforms in April 1991

Design - Prospective observational study of 10, first wave fundholding practices and six non-fundholding practices in Berkshire, Buckinghamshire, Northamptonshire, and Oxfordshire.

Results - NHS outpatient referral rates increased in fundholding practices from $107 \cdot 3$ per 1000 patients per annum in 1990-91 (the preparatory year) to 115.4 in 1993-94. Nonfundholders' rates also increased, from $95 \cdot 0$ to $120 \cdot 5$. Out of district referrals and private referrals decreased in both groups of practices. The number of items prescribed and their costs increased steadily over the three years of the study in all types of practice. Between the first two years of the study costs went up least among fundholders and most among controls but this situation was reversed in the following year.

Conclusion - There was no evidence that budgetary pressures caused first wave fundholders in this study to reduce referral rates and no suggestion as yet that fundholding has encouraged a shift away from specialist care. Evidence from prescribing data is rather more complicated. During the first year of fundholding the scheme seemed to have curbed prescribing costs more effectively than the indicative prescribing scheme for non-fundholders but this effect was not sustained in the longer term.

GENERAL PRACTICE (I)

Assessing the outcome of making it easier to change GP; an analysis of the characteristics of those practices which people move from and to

KATE THOMAS, JON NICHOLL, PAT COLEMAN (Medical Care Research Unit, SCHARR, University of Sheffield)

Background - The government white paper, Promoting Better Health, suggested that primary health care services should be made more responsive to patient needs and that competition, brought about by the freer movement between practices, could act as a mechanism for improving the quality of the services provided.

Aims - The study aimed to estimate the volume of movement between practices not attributable to a change of address, and to investigate which practice characteristics people moved towards and which they moved away from as a result of choosing to change their GP.

Method - Data on 2617 "patient movements" involving no change of address were collected from five family health services authorities (FHSA) during June 1991. These patient movements were analysed in relation to data on practice characteristics obtained from FHSA records.
Results - The estimated volume of movements was small $(1.6 \%$ of the registered population per year). Most movements fulfilling the study criteria were between group practices, a quarter of the movements recorded were to single handed practices. However, the ratio of the number of movements from group practices to single handed practices compared with those from single handed to group practices was 1.37 (95\% confidence interval $1 \cdot 19$, $1.57)$. In choosing single handed practices, these people were willing to forgo access to a female GP, extended services, and greater hours of availability. Among the subset of movements between group practices, people of both sexes were more likely to gain access to a female GP, a practice nurse, and longer surgery hours as a consequence of their move between practices.

Conclusion - It is concluded that the scale of movement observed did not indicate any substantial mechanism by which the new policy of encouraging consumerist behaviour on the part of primary care users could effect desired changes in primary care practice. Among the movements observed, the evidence suggests that potential patients are not deterred by single handedness when choosing a practice and that there may be an underlying conflict between the public's perception of the factors contributing to quality of service and the official characterisation of "good practice" or high quality services in primary health care.

\section{Do general practitioners influence the uptake of breast cancer screening?}

F A MAJEED, ${ }^{1}$ D G COOK, ${ }^{1}$ R GIVEN-WILSON,${ }^{2}$ P veCCHI, ${ }^{2} \mathrm{~J}$ POLONIECKI ${ }^{1}$ ( ${ }^{1}$ Department of Public Health Sciences, St George's Hospital Medical School, London; ${ }^{2}$ South-West London Breast Screening Service)

Objectives - To investigate the relative importance of patient and general practice characteristics in explaining variations between practices in the uptake of breast cancer screening.

Design - Ecological study examining variations in breast cancer screening rates among 131 general practices.

Setting - Merton, Sutton and Wandsworth Family Health Services Authority.

Main outcome measure - Percentage of eligible women aged 50-64 years who attended for mammography during the first round of screening for breast cancer (1991-94).

Results - Of the 43063 women eligible for breast cancer screening, $25826(58 \cdot 2 \%)$ attended for a mammogram. Breast cancer screening rates in individual practices varied from $12 \cdot 5 \%$ to $84.5 \%$. The estimated percentage list inflation for the practices was the variable most highly correlated with screening rates $(r=-0 \cdot 69)$. There were also strong negative correlations between screening rates and variables associated with social deprivation, such as the estimated percentage of the practice population living in households without a car $(r=-0.61)$, and with variables that measured the ethnic make up of practice populations such as the estimated percentage of people in non-white ethnic groups ( $r=$ $-0 \cdot 60)$. Screening rates were significantly higher in practices with a computer than in those without $(59.5 \% v 53.9 \%$, difference $5 \cdot 6 \%, 95 \%$ confidence intervals $1 \cdot 1,10 \cdot 2 \%$ ) In a forward stepwise multiple regression model that explained $58 \%$ of the variation in breast cancer screening rates, four factors were significant independent predictors (at $\mathrm{p}=0.05)$ of screening rates: list inflation and people living in households without a car were both negative predictors of screening rates, and chronic illness and the number of partners in a practice were both positive predictors of screening rates. The practice with the highest screening rate $(84.5 \%)$ contacted all women invited for screening to encourage them to attend for their mammogram and achieved a rate $38 \%$ higher than predicted from the regression model.

Conclusions - The strong negative correlation between breast cancer screening rates and list inflation shows the importance of accurate age-sex registers in achieving high breast cancer screening rates. Breast cancer screening units, family health services authorities, and general practitioners need to collaborate to improve the accuracy of the age-sex registers used to generate invitations for breast cancer screening. The breast cancer screening rate achieved by the practice with the highest screening rate suggests that practices can influence the uptake of breast cancer screening among their patients. Giving general practitioners a greater role in breast cancer screening, either by offering them financial incentives or by giving them clerical support to check prior notification lists and contact non-attenders, may also help to increase breast cancer screening rates.

Costs and remuneration for cervical screening in general practice: are practices in deprived areas penalised?

SUSAN MYLES, ${ }^{1}$ SALLY WYKE, ${ }^{1}$ TRACY IBBOTSON, ${ }^{2}$ SALLY MACINTYRE, ${ }^{3}$ JIM MCEWEN, ${ }^{4}$ MOYA KELLY ${ }^{5}\left({ }^{1}\right.$ Department of General Practice, University of Edinburgh; ${ }^{2}$ Health Services Research Unit, University of Aberdeen; ${ }^{3} M R C$ Medical Sociology Unit, Glasgow; ${ }^{4}$ Department of Public Health, University of Glasgow, ${ }^{5}$ Department of Postgraduate Medicine, University of Glasgow) Objective - To examine the detailed costs of organising cervical screening in general practice in relation to remuneration and deprivation.

Setting - Eighty seven general practices in Greater Glasgow Health Board $(47 \%$ of all practices).

Methods - Detailed costs for the organisation of cervical screening were calculated in terms of who did which tasks and the time they took to do them. These included costs of the call/recall system; doing the smears themselves; dealing with results; and stationary and other material costs. Remuneration for each quarter was calculated based on the "red book" formula, which takes number of smears performed in the practice, uptake and number of partners into account. Remuneration to costs ratios (RCRs) were calculated for each practice. The resulting distribution was not normally distributed but became so after transformation with natural logarithms.

Results - RCRs varied widely between practices. Sixteen per cent of practices did not break even, earning less than they "spent" on the organisation of screening, while $15 \%$ of practices received over $£ 5$ in remuneration for every $£ 1$ "spent". The median RCR was $£ 2.13$ earned for every $£ 1$ "spent". RCRs did not vary by Jarman score (Pearson correlation coefficient $=0.035, p=0.75)$ despite uptake being lower in practices in more deprived areas. RCRs did, however, vary by list size (correlation coefficient $=0.28, p=0.009$ ), and by who took smears (correlation co- 
efficient $=0.63, p=0.0001$ ). RCRs were much lower if general practitioners took smears than if nurses did. Practices with higher Jarman scores were significantly more likely to involve nurses in taking smears than those with lower scores.

Conclusion - General practitioners working in more deprived areas in Greater Glasgow were more likely to have lower uptake rates - often by virtue of relatively intractable practice characteristics. This did not, however, serve to reduce their remuneration compared with their costs of organising screening. This is probably because practices in more deprived areas were much more likely to use nurses to organise and take cervical smears, which constituted a more efficient organisational approach. Cheaper nurse labour costs favourably influenced their RCRs. In addition, skill mix opportunity costs were minimised with practices in more deprived areas freeing up general practitioner time for other tasks.

THIRD WORLD HEALTH

Social inequalities in health status and care practices among patients with diabetes mellitus in Trinidad and Tobago

M C GULLIFORD, S M ARIYANAYAGAM-BAKSH, L BICKRAM, D I PICOU, D MAHABIR ( $\mathrm{Com}$ monwealth Caribbean Medical Research Council; Nutrition Division, Ministry of Health, Trinidad and Tobago; Department of Public Health Medicine, UMDS, St Thomas's Campus, London)

Objective - Chronic non-communicable diseases such as diabetes mellitus are now major contributors to morbidity and health care costs in middle income countries like Trinidad and Tobago. Government health services aim to provide access to basic services for the whole population. This paper examined whether this objective was achieved.

Methods - A register was compiled of all admissions with diabetes to general medical, general surgical, and ophthalmology wards at Port of Spain Hospital between October 1993 and April 1994. A structured interview was conducted which included details of health status and patients' use of health care and self care.

Results - Of 1447 patients admitted to hospital with diabetes, $1149(79 \%)$ responded to a structured interview, $138(12 \%)$ had no schooling, $782(68 \%)$ had primary education only, $199(17 \%)$ had secondary education or higher, and $30(3 \%)$ were not classified. Compared with patients with secondary education, those with no education were older, were more likely to be women, or of Indian subcontinent descent. Patients with no schooling were more likely to have chest pain on effort, a history of stroke or heart attack (odds ratio 3.51 (95\% CI 1.97, 6.24)), sensation of burning or numbness in the feet (OR 1.72 $(1 \cdot 11,2 \cdot 68)$ ), and difficulty with eyesight or blindness (OR $2 \cdot 97(1 \cdot 28,6 \cdot 87)$ ) Variations in the prevalence of neuropathic symptoms, previous heart attack, or blindness were mostly explained by confounding with increasing age, female sex, and Indian ethnic group. Patients with no schooling were more likely to attend a health centre than a private doctor as their usual source of care (OR 4.5 $(2 \cdot 76,7 \cdot 35))$, were less likely to have attended a dietitian (OR $0.53(0.34,0.83)$ ), were more likely to use Benedict's solution and less likely to use test strips for urinalysis, and were less likely to test for blood glucose at home (OR $0.14(0.03,0.61))$. Adjusting for age, gender, and ethnic group did not explain social inequalities in the usual source of care, attendance at a dietitian, or the use of home blood glucose monitoring.

Conclusion - The greatest burden of complications of diabetes was found among those who had the lowest educational attainment. These were people who were most dependent on government health services and who found access to more than the minimum level of services difficult to achieve. The results suggest that government services should be better directed at those with the greatest health needs.

Bhopal: morbidity 10 years after the gas leak. An epidemiological study

S D ACQUILLA, P CULLINAN, R BERTELL for the International Medical Commission on Bhopal (Department of Epidemiology and Public Health, University of Newcastle upon Tyne)

Study objectives - These were, firstly, to examine the existence, nature and extent of persisting health problems attributable to the 1984 gas leak in Bhopal and, secondly, to establish a methodology for future locally initiated studies of the long term health effects of the gas leak.

Method - An epidemiological survey of a representative sample of gas exposed inhabitants of Bhopal was conducted in January 1994. For reference a group of unexposed persons in the same city was also surveyed. Health and exposure questionnaires were administered to 474 persons of whom 74 were also clinically examined. Responses to the questionnaire were analysed according to a measure of individual gas exposure.

Results - As expected, a high proportion of subjects reported respiratory symptoms, with a strong gradient in relation to exposure category. This was not accounted for by differences in smoking, and was consistent with the results of spirometric testing. Ophthalmic symptoms were reported significantly more frequently by subjects in high exposure categories and the results of neurological examination and testing tended to confirm this finding. Although a number of other symptoms were similarly correlated with exposure, with the possible exception of gastrointestinal disease, there was no clear evidence of other organ system damage attributable to the gas exposure.

Conclusion - The methodology of this survey is proposed as a model for low cost epidemiological studies of the long term morbidity among survivors of the 1984 disaster.

Health policy in low income countries: towards the millennium

ANTHONY B ZWI, ANNE MILls (Health Policy Unit, London School of Hygiene and Tropical Medicine)

Health policies worldwide have changed dramatically in recent decades. We reflect upon these changes, highlight current trends, and identify key issues and challenges as the year 2000 approaches. We consider first the context within which health policies in low income countries are formulated and im- plemented: macropolitical and macroeconomic developments, assessment of health needs and their determinants, changing approaches to health planning, priority setting and financing, and dynamic relationships between a number of the key international health policy actors - notably the World Bank and World Health Organization. Next, we provide an overview of the content of current health policy proposals in less developed countries, notably those concerned with the financing, organisation, and management of health systems. Finally, we present some concerns for health policy in the millennium: issues of equity, policy analysis, evidence based policy making, increased accountability, and modified approaches to delivering foreign assistance to the health sector are considered.

AIDS RELATED ISSUES

Mortality in the entire UK haemophilia population before and after HIV infection

DAVID W EWART, ${ }^{1}$ SARAH C DARBY, ${ }^{1}$ PAUL L F GIANGRANDE, ${ }^{2}$ CHARLES $R$ RIZZA, ${ }^{2}$ PAUL J DOLIN, ${ }^{1}$ ROSEMARY J D SPOONER, ${ }^{2}$ on behalf of the UK Haemophilia Centre Directors' Organisation ('Imperial Cancer Research Fund Cancer Epidemiology Unit, University of Oxford; ${ }^{2}$ Oxford Haemophilia Centre, Churchill Hospital, Oxford)

Objective - To describe mortality both before and after the introduction of HIV-1 infection into the complete population of haemophilia patients in the UK

Setting - The UK national haemophilia register. This has included all males living in the UK and diagnosed with haemophilia since 1976.

Patients - A total of 6281 patients was included in the national register in the period 1977-91. During 1979-86, transfusion therapy carried a risk of infection with HIV-1. One fifth of the population became infected, with median seroconversion date October 1982

End points - Mortality.

Main results - Among 2449 patients with severe haemophilia, the age standardised an nual death rate was stable at 8 per 1000 during 1977-84, and during 1985-92 this remained identical at 8 per 1000 among patients not known to be seropositive for HIV. In contrast, the death rate in seropositive patients rose steeply year by year after 1984 reaching 80 per 1000 in 1991-2, a death rate 10 times higher than was seen before the introduction of HIV into the population Among 3832 patients with moderate or mild haemophilia a similar pattern was observed with the death rate among patients tested seropositive for HIV rising to 81 per 1000 in 1991-2. A total of 402 deaths occurred in HIV seropositive patients during 1985-92, whereas only 57 would have been predicted on the basis of rates in patients not tested seropositive. Most of the excess deaths were certified as due to AIDS or to condition recognised as being associated with AIDS. Conclusions - These are the first data to document that in a large and complete population mortality among those who are not infected with HIV has remained unchanged over time, while mortality has increased greatly in those who became infected. 
Characteristics of injecting drug users classified by whether they reported ever receiving used injecting equipment

\begin{tabular}{llllll}
\hline & $\begin{array}{l}\text { HIV }+v e \\
(\%)\end{array}$ & $\begin{array}{l}\text { HBc }+v e \\
(\%)\end{array}$ & $\begin{array}{l}\text { Median } \\
\text { age }(y)\end{array}$ & $\begin{array}{l}\text { Female } \\
(\%)\end{array}$ & $\begin{array}{l}\text { Median first } \\
\text { year of injection }\end{array}$ \\
\hline Ever & $38 / 1965(1 \cdot 9)$ & $754 / 1965(38)$ & 29 & $564 / 1958(29)$ & 1985 \\
Never & $3 / 1252(0 \cdot 2)$ & $332 / 1248(27)$ & 27 & $294 / 1247(24)$ & 1988 \\
\hline
\end{tabular}

Aids and infection with HIV among UK adolescents

JONATHAN ELFORD, ${ }^{1}$ MELANIE WEERASURIA, AHILYA NOONE ${ }^{2}\left({ }^{1}\right.$ University Department of Public Health, Royal Free Hospitai School of Medicine, London; ${ }^{2}$ PHLS Communicable Disease Surveillance Centre, London)

Objective - To examine the epidemiological characteristics of AIDS and infection with HIV among adolescents in the United Kingdom.

Method - Analysis of AIDS surveillance data. Subjects - Adolescents aged 13-19 years and adults aged $20-29$ years.

Setting - United Kingdom.

Subjects - All those diagnosed with AIDS and reported to the CDSC and the Scottish Centre for Infection and Environmental Health by 31 December 1994.

Results - By 31 December 1994, 10304 cases of AIDS had been reported in the United Kingdom (9414 males, 890 females). Of these, $68(0.7 \%)$ cases were in adolescents aged 13-19 years (59 males, 9 females). A further $2179(21 \cdot 1 \%)$ AIDS cases were diagnosed in young adults aged $20-29$ years (1832 males, 347 females) many of whom would have been infected with HIV in adolescence, given the prolonged period between infection with HIV and the development of major symptoms. The percentage of females with AIDS who were diagnosed between 13-29 years $(40.0 \%, 356 / 890)$ was twice that seen in males $(20 \cdot 1 \%, 1891 / 9414)$. Among males and females aged 13-29 years at AIDS diagnosis whose exposure to HIV was behaviour related, sexual intercourse accounted for greater proportion of cases than injecting drug use (males $82.9 v 8.1 \%$, females $76.1 \%$ v $19.4 \%$ respectively). However, of those people aged 13-29 years at AIDS diagnosis who acquired HIV through heterosexual contact, females $(n=271)$ outnumbered males $(n=155)$ by almost two to one. Over the age of 30 years this pattern was reversed. Males diagnosed with AIDS at age 13-29 years who acquired HIV through sexual contact ( $n=$ 1568) were more likely to report having had sex with another man $(90.1 \%, 1413 / 1568)$ than with a woman only $(9.9 \%, 155 / 1568)$ The percentage of males who reported having sex with another man declined from $97 \%$ in 1985 to $83 \%$ in 1993 .

Conclusion - AIDS surveillance data suggest that females with AIDS were more likely to have been infected during adolescence than males. For adolescent males, sex between men seemed to account for most cases of sexually acquired HIV infection. These findings have important policy and legislative implications for the health of adolescents.

Questioning the assumption that all injecting drug users share needles and syringes

P MADDEN, A DURANTE, D BENNETT (PHLS AIDS Centre, PHLS Communicable Disease Surveillance Centre, London)

Background - Injecting drug users (IDUs) are identified as a group at high risk of HIV infection based on the assumption that most of them share needles and syringes. The proportion of injectors who have shared injecting equipment has important implications for HIV transmission. The identification of groups of IDUs at increased risk of ever having shared is important for planning and evaluating HIV prevention.

Methods - The Public Health Laboratory Service unlinked anonymous survey of HIV infection in IDUs has monitored the prevalence of salivary antibody to HIV and hepatitis B core $(\mathrm{HBc})$ and recent risk behaviours in current and past injectors attending drug agencies since 1990 . In 1993, 3411 subjects at 35 centres were asked if they had ever received used needles or syringes from anyone.

Results - HIV prevalence was low and unchanging (1-2\%) between 1990 and 1993 . Thirty nine per cent (1252/3217) of subjects in 1993 reported never receiving used injecting equipment. Their characteristics are compared with those who had received used equipment in the table above.

Discussion - The high proportion of subjects who reported never receiving used needles and syringes indicates that many IDUs in England and Wales may never have been at risk of HIV infection from their injecting drug use. The prevalence of HIV among those who never shared may represent sexual transmission. These data suggest that those who began to inject more recently are less likely to have ever received syringes. The independen lihood of ever sharing will be assessed with logistic regression.

\section{Life support training in the first year of}

C A LESTER, P D DONNELly (Centre for Applied Public Health Medicine, University of Wales College of Medicine, Cardiff)

Objective - To investigate the feasibility of teaching cardiopulmonary resuscitation (CPR) in the first year of secondary school, and the effect of pupil instructors (peer tutoring) on skills and self perception of ability. Method - Of nine first year mixed ability classes (age range 11-12 years) comprising 253 boys and girls, four classes were instructed in CPR by the teacher only and five by the teacher assisted by older pupils. Al trainers were qualified CPR instructors. Before training each child completed a registration form collecting demographic details and information on previous CPR training and known family members with a heart problem. Post training pupils were tested in practical skills by a senior pupil or teacher and completed questionnaires on CPR theory, attitude, and willingness to perform in an emergency. effects of subject characteristics on the like-

CARDIOVASCULAR DISEASE MANAGEMENT (I) secondary school
Results - One pupil was opted out by a parent, one refused to take part, and two were withdrawn by a teacher: a further 10 were not included as they were absent for more than one lesson. This left 239 pupils - 100 instructed by the teacher only and 139 by the teacher and older pupils. There were 115 $(48 \%)$ who were aware of one or more relatives with a heart problem, most commonly a grandfather. Eighty seven (36\%) had some previous knowledge of CPR, usually from scouts or guides. Post instruction skill testing showed no significant difference between those taught by the two methods but $81 \%$ of girls achieved marks of $90 \%$ or more compared with only $59 \%$ of boys $(p<0.001)$. The pupils' belief in their own ability to save a life in an emergency followed a similar pattern with $73 \%$ of girls believing themselves capable compared with only $53 \%$ of boys $(p<0.001$ ) Those instructed by the teacher only showed greater confidence in their abilities with $70 \%$ who thought themselves capable of saving a life, compared with $56 \%$ instructed by teachers and older pupils $(p<0 \cdot 025)$. Overall, 92\% believed that everyone capable should be instructed in CPR. The most frequently mentioned circumstances in which pupils would be reluctant or unwilling to resuscitate were the presence of vomit, bleeding from the mouth or face, severe injuries or profuse bleeding, or a casualty who looked dirty or smelt unpleasant.

Conclusion - It is feasible to teach CPR to children in the first year of secondary school but effectiveness does not seem to be enhanced by peer tutoring, which may be detrimental to confidence.

A multicentre randomised controlled trial of rehabilitation after myocardial infarction

D A JONES, R R WEST (University of Wales College of Medicine, Cardiff)

A role for rehabilitation after myocardial infarction (MI) is now widely recognised and many new programmes are being established in the UK. However, many programmes are predominantly exercise based and offered only to younger patients and their effectiveness is demonstrated only for younger and male patients or volunteers, and mostly in small trials and in other countries.

This paper reports the evaluation of rehabilitation in unselected patients by means of a multicentre randomised controlled trial. Rehabilitation programmes were set up in six district general hospitals for MI patients (and spouses), comprising psychological therapy, counselling, relaxation training, and teaching stress management over seven weekly group outpatient sessions. All patients admitted over two years with confirmed $\mathrm{MI}$ and discharged home within 28 days were eligible (2287 patients). Data were collected (i) on admission, (ii) by structured interview of patients (and spouses) shortly after discharge, (iii) again by structured interview at six months, and (iv) by clinical examination at 12 months.

At six months, rehabilitation patients reported reduced frequency of (i) angina $(6.0$ $v 6.9$ per wk) (ii) angina medication $(7.8 v$ 8.9 uses per wk), and (iii) medication generally (3.6 $v 3.8$ prescriptions). There were no significant differences between rehabilitation patients and controls in reported anxiety (prevalence $32 \%$ ) or depression (19\%), clinical 
complications, and clinical sequelae, and only a small difference in early mortality at six months which was balanced within 12 months. Rehabilitation programmes for unselected MI patients based on psychological therapy, counselling, relaxation training, and stress management seem to offer modest benefit compared with previous reports of smaller trials of intensive programmes offered to selected younger men or volunteers. While objective benefits were modest, patients (and spouses) reported satisfaction and considered that programmes had aided rehabilitation.

\section{Stroke care in Europe - can we learn lessons from the different ways stroke is managed in different countries?}

M RATCLIFFe, R BEECH, C WOLFE (Department of Public Health Medicine, UMDS, St Thomas's Campus, London)

Background - Stroke is the third most common cause of death in Europe after cancer and heart disease, accounting for $10-12 \%$ of all deaths in industrialised countries. It has been estimated that one million acute ischaemic strokes occur in Europe each year. The SMR varies from 78 in France to 199 in Portugal, with age standardised mortality rates of 33.5/100 000 in Portugal. In spite of these statistics there is a lack of knowledge of the reasons why mortality from stroke varies between countries.

Objective - To explore the variation in the clinical management of stroke and the resource implication of this variation as related to outcome.

Design - The study has a cross sectional design. The data are gathered in 22 centres in eight different EU countries. Case severity is established within 48 hours of stroke for all stroke patients admitted to each centre. Patients are then followed up at three and 12 months, to record all inpatient and outpatient clinical activity and resource input. Economic evaluation of each centre is undertaken to establish cost.

Results The study is now in its 18 th month so we have reliable follow up data on $50 \%$ of all patients admitted to the study in the first year. These preliminary data are already showing wide variation in approaches to the clinical management of patients across variables such as type of bed admitted to, the amount of inpatient rehabilitation received, and the number and type of diagnostic techniques used. One of the German centres completed brain imaging on $94 \%$ of all patients with a further $36 \%$ receiving angiography, $80 \%$ receiving Doppler, and $40 \%$ receiving echocardiagram. This compares with $30 \%$ receiving brain imaging, a further $1 \%$ receiving angiography, and no patient receiving Doppler or echocardiagram in one of the UK centres. Similarly, bed type usage shows wide variation in Spain and Germany where $81 \%$ and $48 \%$ respectively of all patients are admitted to neurology beds. In the UK this figure is only $1.5 \%$ of all patients. The economic evaluation is also showing wide variation in the amount of doctors' and nurses' time devoted to both direct and indirect patient care.

Conclusion - All the results are set within the context of countries' systems of health planning, policy, and finance and highlight, perhaps for the first time, real links which exist between health policy and patients' treatment and outcome at a disease specific level.
ISSUES AROUND PUBLIC HEALTH MEDICINE (CHAIR'S SESSION)

The evidence for the effectiveness of NHS interventions to reduce inequalities in health

$M$ LAMBERT, L ARBLASTER, $\mathrm{V}$ ENTWISTLE, $\mathrm{T}$ SHELDON, I WATT (NHS Centre for Reviews and Dissemination, University of York

Introduction - Many of the determinants of inequalities in health lie outside the direct control of health services but it is possible that appropriate health services interventions can, to some extent, reduce these inequities. In 1994 a review of such interventions was commissioned by the Department of Health. Objectives - To review published reports to dentify evaluations of interventions which the NHS, alone or in collaboration with other agencies, could use to reduce inequalities in health, particularly those inequalities relating to the Health of the Nation areas of coronary heart disease and stroke, cancers, mental illness, HIV/AIDS and sexual health, and accidents

Methods - A systematic literature review of experimental and quasi-experimental studies of interventions which set out to improve poor health associated with differences in socioeconomic status, or with ethnicity, was instituted. Interested professionals, academics, and relevant organisations were contacted for information. Database and literature sources were searched. All citations and material obtained were assessed for relevance and all studies included were critically appraised according to agreed criteria. Studies which were judged by the reviewers to provide reasonable evidence of an intervention's success were analysed to identify common characteristics.

Results - From the large number of published and unpublished reports of health service based interventions, 82 reports of evaluated interventions which met the inclusion criteria were identified, of which 18 were carried out in the UK. Thirty of the studies were randomised controlled trials. Most of the studies identified had significant methodological flaws. A checklist for use by professionals designing interventions to improve health of deprived populations is developed from studies which provide reasonable or good evidence of effectiveness.

Conclusions - A number of health service based interventions directed at disadvantaged groups so as to improve their health have been evaluated. Some have been shown to be effective. Identifying relevant evaluations is difficult because of the wide variety of professional disciplines involved and the lack of consistency of terminology. The extent to which the identified interventions might reduce inequalities in health is yet to be identified.

\section{The audit cycle - myth or reality?}

MARTIN ECCLES, ELAINE MCCOLL, MARK DEVERILL, HELEN RICHARDSON (Centre for Health Services Research, University of Newcastle upon Tyne)

Aims - Medical audit is increasingly part of medical practice, both in primary care and in the hospital setting, and is intended as a means of quality improvement. To this end, an important part of the audit cycle is the identification of opportunities for change and the implementation of that change. But doubts have been expressed as to whether audit is effective in quality assurance; it has been suggested that some participants see it primarily as an educational activity. As part of a large study of audit across the primarysecondary care interface, we set out to examine the extent to which those engaged in audit actually managed to "close the loop" by identifying the need for change and implementing that change.

Methods - The interface audit survey was a three part postal survey using a cascade sampling approach. The second ques tionnaire asked for factual details of the audit, including information on whether each step of the audit cycle had been completed. In addition, respondents were asked to indicate whether they considered the audit to be complete at the time of response. The third questionnaire sought respondents' views of the experience of audit.

Results - Data on 379 interface audits were obtained. Of those deemed to be "complete", only $10 \%$ had in fact carried out all nine steps in the audit cycle. Seventy nine per cent had identified the need for change, but only $40 \%$ had tried to implement change. The percentages evaluating change and re-auditing were even lower at 22 and $19 \%$ respectively. Nonetheless, most respondents had very positive views of the audit process, feeling that it offered opportunities for interprofessional collaboration and stimulated learning.

Discussion - The results of this survey are disturbing. Medical audit is intended as a mechanism for quality assurance. But a substantial minority of respondents who claimed to have completed their audit had not even identified the need for change, while less than half had attempted to implement change. Thus, while audit may be succeeding as an educational activity and as a basis for professional development and collaboration, our findings suggests that it may be failing in the prime objective of improving quality of care This raises important questions about the cost-benefit relationship in the audit process

\section{The training and career development} needs of public health professionals

L SOMMERVAILLE, R GRIFFITHS (Department of Public Health and Epidemiology, University of Birmingham)

The Faculty of Public Health Medicine currently operates as a standard setting body for the training and career development needs of public health physicians. As part of the debate as to whether it should include other professionals under its umbrella, it commissioned a survey of non-medical public health specialists in England, Wales, and Scotland. This was a previously undefined group, and in order to make contact with as many professionals as possible, initial contact was made via the employing organisations.

The study was carried out as a short posta survey collecting information on academic and professional qualifications, identification of training needs, and career hurdles and aspirations. A total of 1520 questionnaires were sent out and of the 1072 returned, 979 were included in the database. Survey respondents were categorised as belonging to either service or academic organisations.

In the group as a whole, 706 individuals were qualified to first degree level, 500 to masters degree level, and 195 had a PhD. Some 339 respondents had either nursing, 
teaching, or management qualifications However, nearly $50 \%$ of the respondents felt that they had received no formal training for their current job. In terms of career as pirations, $72 \%$ of the respondents said they would like to remain in public health but $54 \%$ said there was no structure in thei present organisation to allow them to develop a career. The main hurdles cited as blocks to career development were the domination of public health by the medical model and the lack of both training and career development structures. For the academic group, $23 \%$ also felt that short term contracts and the un certainties of funding were a major problem Isolation also seems to be common in this group. When asked to list memberships of any relevant professional bodies 656 respondents, $74 \%$ of the service group and $54 \%$ of the academic group, did not record any at all.

It is certain that the survey respondents do not represent all non-medical public health practitioners in the UK but they do represen a sizeable, knowledgeable, and experienced group which is not being invested in by the NHS.

\section{What impact do annual public health reports have?}

NAOMI FULOP, MARTIN MCKEE (London School of Hygiene and Tropical Medicine)

Background - Annual public health reports on the health status of local populations were reintroduced following the recommendations of the Acheson Report in 1988. This population based approach became more pertinen with the introduction of the NHS reforms in 1991 whereby district health authorities were given the responsibility of purchasing health services for their resident population. A number of studies have reviewed their style and content; suggestions have been made for timetables and production; and attempts have been made to define criteria for audit. However, very little evidence has been collected on the impact of these reports.

Objective - To evaluate the impact of annual public health reports.

Design - This was a questionnaire survey in two stages. In stage one, questionnaires were sent to 13 directors of public health and copies of their last report were requested. In stage two, questionnaires and reports (where available) were sent to 126 senior health social services, and environmental managers in one regional health authority. Questionnaires asked for their views on the impact of their local report.

Setting - The 13 district health authorities in North Thames Regional Health Authority.

Results - The response rate for directors of public health was $92 \%$. A preliminary analysis shows that all bar one report contained recommendations which resulted in changes in purchasing of services. All bar one report had attracted media attention - mainly local newspapers and radio. Less than half $(46 \%)$ of reports had been discussed at local authority committees - mainly social services and housing. Initial results from stage two indicate difficulties in aiming reports at a broad audience, including lay people. Improvements suggested included: that reports should be more integrated with overall health authority strategy; they should contain an evaluation of the impact of changes in commissioning. and they should use more accessible language and graphics.

Discussion - The production of annual public health reports requires a significant level of resources so it is important to analyse their impact. The results from both stages of the survey will be discussed in relation to join working between health and local authorities and the move to primary care-led commissioning.

Casemix measurement in the NHS internal market - an evaluation of available approaches

NEIL SÖDERLUND, ${ }^{1}$ ALASTAIR GRAY, ${ }^{2}$ JAMES RAFTERY, ${ }^{3}$ RUAIRIDH MILNE ${ }^{1}\left({ }^{1}\right.$ Department of Public Health and Primary Care, University of Oxford ${ }^{2}$ Centre For Socio Legal Studies, Wolfson College, University of Oxford; ${ }^{3}$ National Casemix Office, Winchester)

Background - The NHS internal market has highlighted the importance of developing appropriate, valid, and timely measures of hospital activity, both for the purposes of specifying and monitoring contracts and for evaluating the success of the NHS reforms in general. Both imported and local casemix systems are now available in the United King dom, the best known examples of which are diagnosed related groups (DRGs) and healthcare resource groups (HRGs) respectively. Despite the recommendation of HRGs as a "language for contracting" by the NHS Executive, no formal analysis has compared available grouping approaches. This paper compares the predictive validity of five easily accessible approaches to casemix classification, DRGs, HRGs versions 1 and 2 , specialty classification, and a simple age categorisation.

Methods - Two data sets were used to compare different approaches to casemix. A 3\% random sample $(n \simeq 300000)$ of the 1992-93 hospital episodes statistics (HES) was used to test their ability to predict variation in the length of stay (LOS), and a second set of individually costed patient episodes from two hospitals $(n \simeq 80000)$ was used to test their ability to explain cost variation. Each was tested using untrimmed data, and data trimmed at $\operatorname{LOS}=100$ and $\operatorname{LOS}=30$ days . Analysis of variance models were used to assess the fit of each of the casemix systems to test data, and a simple significance test of differences in mean squared error between models was applied.

Results - All casemix methods performed poorly on untrimmed data. When lengths of stay greater than 29 days were excluded, version 2 of HRGs explained $31 \%$ of total variance in $L O S$ and $25 \%$ of cost variation, and fitted the data significantly better than any of the other options $(p<0.001)$. DRGs explained less variance than did version 2 of HRGs but performed better than HRGs version 1 . This pattern was generally borne out for specialty specific analyses as well, although DRGs were superior in paediatrics, surgical subspecialties, and geriatrics in at least one of the data sets. HRGs and DRGs seemed to be more valuable in surgical specialties, explaining 51 and $44 \%$ respectively of the variance in LOS for gynaecology, for example. At the other extreme, reductions in variance for medicine, geriatrics, and psychiatry were less than $20 \%$ for all casemix systems.

Conclusions - The healthcare resource groups (HRG) casemix system seems to offer better performance in terms of resource homogeneity than other currently available approaches. It is also substantially cheaper than imported alternatives and has been formally endorsed by the royal colleges. In applying any casemix system to contracting or research applications, care should be taken with exclusion of outlying data, different methods for which yield substantially different results.

Local NHS health care purchasing and prioritising from the perspective of Bromley residents - a qualitative study

J BARKER (Department of Public Health, Bromley Health, Kent)

Aim - To inform the development of a model for involving local people in planning and prioritising of health care.

Objectives - To identify levels of awareness and views of the NHS reforms and Bromley Health's role in the provision of local health care. To explore reactions to the concept of a finite budget and the need to prioritise health care. To explore the value of providing cameos of specific purchasing dilemmas to generate discussion and debate of the process of prioritisation within the context of effectiveness, appropriateness, cost, value for money, and finally values/perceived importance. To explore the perceived role of the public in this process. To elicit views on the changing face of health care using specific examples of day case surgery, shift of services from acute to primary care, and care of the mentally ill in the community.

Design - This was a qualitative study using six in depth discussion groups made up of local residents recruited by an agency to reflect the sociodemographic characteristics of Bromley. Each group contained up to nine respondents of the same sex and age group. Three age groups were used (18-30 years, 35-50 years, 55-70 years). Each group discussion lasted one and a half hours and was taped and transcribed.

Results - There was virtually no awareness of what Bromley Health is or does. The reforms were perceived to be a series of ways of saving money. This negative view seemed to act as a filter through which all information about health care passed. A finite budget and prioritising were seen to be acceptable and necessary. Faced with purchasing dilemmas people were less likely to consider exclusion of services as a good idea. Groups clearly appreciated that conflict of values was a major problem but expressed the view that the public should not be part of a formal decision making process - this should be left to managers and medical staff. It was felt, however, that the public should have an opportunity to have its views heard. Public meetings were not seen to be a useful way of eliciting views and consulting.

Conclusions - There is a need to develop an interactive model for involving the public which will both inform and raise awareness as well as capture views and values on the issues around prioritising. PART - the public awareness raising tool - has been developed in Bromley and informed by the recommendations of this research. PART is a key organisational objective for 1995-6.

Infectious disease control in managed markets

J A ROBERTS (Health Services Research Unit, Department of Public Health and Policy, London School of Hygiene and Tropical Medicine)

Objectives - To explore the economic implications of managing infectious disease control programmes in managed markets. To 
model the geographical and time distribution of risk. Reference will be made to hepatitis A, B, and C; Escherichia coli 0157; MRSA; and food borne infections.

Design - The paper will draw on economic analysis, including the economics of managed markets, and previous economic evaluative studies undertaken by the author to illustrate the geographical and time dimensions of the impact of outbreaks upon health services and the community. The analysis will include the use of modelling techniques, using Markov chain type models, and will include hazard analysis, using the HACCP approach. The implications of infectious disease for purchasers and providers of health care in the reformed system will be explored. Case studies will be used to demonstrate the possible economic impact of an outbreak of MRSA and $E$ coli on providers of services; the impact of intervention strategies to reduce Hepatitis $B$ and $C$ infections on individuals and future providers of services; and the impact of prophylaxis for hepatitis $A$ and malaria on the distribution of costs to the health sector and individuals.

Results - The analysis will demonstrate how risks might be distributed between institutions, areas, and in time. The economic impact of primary, secondary, and tertiary prevention will be indicated and the interdependence of the decisions of the various purchasers and providers about infectious disease treatment and control will be modelled. Conclusions - The need to develop a suitable regulatory framework of guidance for purchasers and providers who are involved in contracting for care of persons with infectious disease and for infectious disease control programmes will be indicated. The immature managed market that is emerging in the reformed NHS does not yet seem to have dealt adequately with these classic issues that pose problems for public health and economic analysis.

PREGNANCY AND CHILDHOOD (I)

Importance of motivational factors in health technology assessment: termination of pregnancy study

J MOLlison, s A NAJI (Health Services Research Unit, University of Aberdeen)

Objectives - It is widely accepted that a randomised controlled trial is the most reliable method for conducting clinical research. It is clear, however, that for some interventions, motivational factors will be significant mediators of impact. The patient centred, partially randomised trial design proposed by Brewin and Bradley allows for motivational factors to be equalised in randomised groups and optimised in preference groups. This allows a true treatment effect to be estimated using the two randomised groups but also allows for the independent effect of patients' preferences to be investigated. In a comparison of medical abortion with vacuum aspiration it was anticipated that women's preferences would play an important role in the relative acceptability of these two methods of abortion.

Design - A patient centred, partially randomised trial design was adopted. Women with a strong preference for one method of termination were allocated to that method Women who had no strong preference for either method were allocated at random.

Results - There was no significant difference in the acceptability of procedures among women who were allocated according to their preference. Only three $(4 \%)$ women in either group would opt for a different method in the future. In comparison, women who were allocated at random, and who were at more than 50 days' gestation found vacuum aspiration to be more acceptable. For those at less than 50 days, there were no significant differences. Two (2\%) women randomised to vacuum aspiration would undergo a different method in the future compared with $21(22 \%)$ women randomised to medical abortion.

Conclusions - Women who would prefer a particular method of abortion should receive that method, whereas women who are not sure which method to use should be advised that vacuum aspiration is likely to be more acceptable if they are at more than $\mathbf{5 0}$ days' gestation. These conclusions could not have been reached if a conventional randomised controlled trial had been adopted.

Preventing sudden infant deaths - a test of national public health systems

MARTIN MCKEE, ${ }^{1}$ NAOMI FULAP, ${ }^{1}$ FERENC BOJAN, ${ }^{2}$ JOHN-PAUL VADER, ${ }^{3}$ PAUL BOUVIER, ${ }^{3}$ FINN RASMUSSEN, ${ }^{4}$ LENNART KOHLER, ${ }^{5}$ HELMUT BRAND, ${ }^{6}$ ANGELA HORT ${ }^{6}$ ( ${ }^{1}$ London School of Hygiene and Tropical Medicine; ${ }^{2}$ Medical University of Debrecen, Hungary; ${ }^{3}$ IUMSP, Lausanne, Switzerland; ${ }^{4}$ Uppsala, Sweden; ${ }^{5}$ Nordic School of Public Health; ${ }^{6}$ Hamburg)

Background - The sudden infant death syndrome (SIDS) is among the leading causes of post-neonatal mortality in industrialised countries. Recent research has highlighted that many of these deaths are avoidable by adopting a few simple precautions. These include sleeping in the supine position, avoiding exposure to tobacco smoke, breast feeding where possible, and avoiding over heating. Several countries, including The Netherlands (1987), Norway, Australia, New Zealand and the United Kingdom (1990), Switzerland (1993), and Israel (1994) have introduced national campaigns to promote these messages, with dramatic results.

Methods and results - The paper contrasts the trends in post-neonatal mortality in Hungary,
England and Wales, Denmark, Norway, Sweden, and Switzerland since 1987 (table). The British campaign, involving the voluntary sector and the Department of Health, was launched in November 1991. Together with the growing awareness of the risk factors that preceded the campaign, there has been a decrease of almost $50 \%$ in post-neonatal deaths since 1987. Similar findings are seen in Norway. There has been no such campaign in Hungary, Germany, or Denmark and that in Switzerland only began in 1993. In these countries there has been correspondingly little reduction in deaths, except in the year that the campaign began in Switzerland. In Sweden, advice was given to health workers in 1992 but there was no official public campaign. There was, however, widespread press coverage and there has been a subsequent decline in deaths, albeit from an already low level.

Discussion - The results of research on the causes of SIDS are unequivocal and the costs of implementation are minimal compared with other health care interventions. This information is now widely available in the international literature. National public health authorities have a responsibility to keep up to date with developments such as this. The extent to which they respond to clear, research based evidence that can save lives at low cost is a measure of their effectiveness. The paper will draw on data from other countries not yet to hand and information on public health structures to examine the factors determining why some governments act on available scientific evidence and others do not.

Change in prevalence of risk factors for sudden infant death syndrome between 1991 and 1995

J BUDD, ${ }^{1}$ A ESMAIL, ${ }^{2}$ D JONES ${ }^{1}$ ( ${ }^{1}$ Department of Public Health and Epidemiology, University of Leicester; ${ }^{2}$ Department of General Practice, University of Manchester)

Objective - Although the decline in SIDS mortality has been associated with a decrease in the prevalence of prone sleeping, this decrease has occurred against a background of declining mortality since 1988 . This study aims to examine the change in prevalence of risk factors associated with SIDS as a result of the government's intervention campaign, to examine the extent to which parents have become aware of the campaign messages, and how this has occurred.

Design - This was a prospective postal questionnaire study of 430 parents from three distinct geographical areas in the UK between 1993 and 1995. Questions were asked on behaviour regarding infant care practices, and information relevant to outcome and process evaluation of the government's campaign was sought. Comparison with a study carried out before the intervention campaign provided

Post-neonatal mortality rate (per 1000 live births) and \% of 1987 value

\begin{tabular}{|c|c|c|c|c|c|c|c|}
\hline Year & Hungary & Switzerland & Denmark & Sweden & Germany & Norway & $\begin{array}{l}\text { England } \\
\text { and Wales }\end{array}$ \\
\hline $\begin{array}{l}1987 \\
1988 \\
1989 \\
1990 \\
1991 \\
1992 \\
1993\end{array}$ & $\begin{array}{l}4.34(100 \%) \\
4.02(92 \cdot 6 \%) \\
4.04(93.1 \%) \\
3.99(91.9 \%) \\
4.26(98 \cdot 2 \%) \\
3.85(88 \cdot 7 \%) \\
3.87(89 \cdot 2 \%)\end{array}$ & $\begin{array}{l}2.60(100 \%) \\
2.63(101 \%) \\
2.91(111 \cdot 8 \%) \\
3.05(117.3 \%) \\
2.61(100 \cdot 4 \%) \\
2.47(95 \%) \\
2.08(80 \%)\end{array}$ & $\begin{array}{l}3.5(100 \%) \\
3.0(86 \%) \\
3.3(94 \%) \\
2.9(83 \%) \\
3.0(86 \%) \\
2.4(69 \%)\end{array}$ & $\begin{array}{l}2 \cdot 24(100 \%) \\
2 \cdot 12(95 \%) \\
2 \cdot 11(94 \cdot 5 \%) \\
2 \cdot 46(110 \cdot 1 \%) \\
2 \cdot 60(116 \cdot 4 \%) \\
2 \cdot 10(95 \%) \\
1 \cdot 73(77 \cdot 4 \%)\end{array}$ & $\begin{array}{l}3.65(100 \%) \\
3.51(96 \%) \\
3.45(94 \cdot 5 \%) \\
3.44(94 \cdot 2 \%) \\
3.39(92.9 \%) \\
2.78(76 \cdot 1 \%) \\
2.71(74 \cdot 2 \%)\end{array}$ & $\begin{array}{l}3.7(100 \%) \\
3.8(103 \%) \\
4.0(108 \%) \\
3.1(84 \%) \\
2.7(73 \%) \\
2 \cdot 1(57 \%)\end{array}$ & $\begin{array}{l}4 \cdot 1(100 \%) \\
4 \cdot 1(100 \%) \\
3.7(90 \cdot 2 \%) \\
3.3(80 \cdot 5 \%) \\
3.0(73 \cdot 2 \%) \\
2.3(56 \cdot 1 \%) \\
2 \cdot 1(51 \cdot 2 \%)\end{array}$ \\
\hline
\end{tabular}

Sources: National statistical reports. 
estimates of the behaviour changes that had occurred during this period.

Results - Sixty per cent of babies are laid to sleep on their back and $32.5 \%$ on their sides. Only 3\% are routinely laid on their front. This compares with nearly $26 \%$ of babies who were placed in the prone position before the intervention campaign. Twenty six per cent of parents identified the avoidance of SIDS as a reason for their behaviour and $14 \%$ of parents were following specific instructions. There seems to be little change in the prevalence of smoking and breast feeding as a result of the campaign. Results on what was perceived to be the most important medium for health education will also be presented.

Conclusion - There has been a significant change in parental behaviour resulting from the intervention campaign with the greatest change occurring for sleep position. The message on reduction of smoking has not been as effective. The avoidance of SIDS was an important reason for the change in behaviour.

Family credit families and uptake of school meals in primary school

S M SOMERVILLE, R J RONA, S CHINN, S QURESHI (Department of Public Health Medicine, UMDS, St Thomas's Campus, London)

Background and objectives - To examine the uptake of school meals by families in receipt of family credit over time in relation to the effect of the 1986 Social Security Act, which came into force in April 1988 and which removed their right to free school meals. These families were compensated by an increase in family credit of 65 per school day per child which was the average cost of a school meal in 1988. This was subsequently uprated by the retail price index. Families on income support continued to be entitled to free school meals.

Design - A nutritional monitoring system of mixed longitudinal design.

Subjects - Two samples, each consisting of about 6000 English primary school children; one consisted of "inner city" children with a high proportion of African/Afro-Caribbean and Indian/Pakistani origin, and the other was more representative of the whole population. Each sample was surveyed biennially. Setting - Forty two study areas in England. Outcome measures - The uptake of school meals by family credit children was compared with the uptake of income support children, and of families not on these benefits. The prices of school meals in the study areas were compared with the family credit allowance for school meals.

Results - The change in legislation resulted in an immediate drop in uptake of school meals by family credit children of around $30 \%$ in both samples. In relation to ethnic group, $42 \%$ of the Asian family credit children changed from school meals compared with $18 \%$ of the Afro-Caribbean children. The decline in uptake of school meals by family credit families has continued, except for the Asian family credit children whose uptake increased slightly in 1993. Income support children continued to have high uptake of school meals, of about $90 \%$. By the early $1990 \mathrm{~s}$, half of the family credit children were not taking school meals, and the price of school meals was more than the family credit school meals allowance in $81 \%$ of the study areas in the representative sample, and in $45 \%$ of the inner city areas.
Conclusion - Reinstatement of free school meals while maintaining benefit levels would free the choice of a school meal from the vagaries of area pricing policies and the demands of a strict household budget.

\section{MORTALITY}

\section{European Community study of "avoid-} able" mortality 1980-89

C WOLFE, A P FITZGERALD, S PHILLIPS, $W$ w Holland (Department of Public Health Medicine, UMDS, St Thomas's Campus, London)

Objectives - (1) To highlight the variation in rates of "avoidable" mortality both within and between member countries. (2) To ilustrate the change in mortality rates over time. (3) To identify high risk areas and so promote further research at a local level to investigate deficiencies in the delivery of health care.

Methods - The study was carried out by the EC Working Group on Health Services and "Avoidable Deaths" which has previously considered "avoidable" mortality for the periods of 1974-78 and 1980-84 (EC Atlases of Avoidable Death. 1st and 2nd eds). Each of the EC countries collected mortality data from its constituent administrative health areas. Six teen selected conditions for which mortality should be avoidable, within selected age limits, were considered. Mortality rates in the 368 administrative areas, over the period 1985-89, were compared with national and EC standards using indirectly standardised mortality ratios (SMRs). The annual percentage change in age standardised mortality rates from 1980-84 to 1985-89, was calculated. Between area variations in mortality rates and in the percentage change in mortality were presented in map and tabular format.

Results - EC mortality rates declined for all the conditions studied, with the exception of asthma and breast cancer for which there was a slight increase. The annual percentage declines were $5.9 \%$ for peptic ulcers (age $5-44$ years) and $12.9 \%$ for chronic rheumatic heart disease (ages 5-44 years) whereas all cause mortality (ages $5-64$ years) fell by $2 \%$ annually. For most of the conditions studied there was considerable variation in mortality rates both within and between counties. Th highest national rates for breast cancer mor tality occurred in the UK and Ireland (national SMRs greater than 125). Within England and Wales there was a twofold variation in mortality between district health authorities. National mortality rates from ischaemic heart disease (ages 35-64 years) varied from 45 per 100000 in France to 219 per 100000 in Scotland.

Conclusions - The observed variations in mortality are not necessarily due solely to variations in health care efficacy. Variation may be partly an artefact of inconsistencies in the diagnosis, classification, and coding of deaths but misclassification does not fully explain the observed levels of variation. For conditions such as asthma and ischaemic heart disease, for which not all deaths are truly avoidable, they may reflect a difference in morbidity levels, but for conditions such as tuberculosis increased morbidity levels indicate deficiencies in the availability and targeting of primary care. The rapid fall in "avoidable" mortality provides indirect evidence for an association with the quality of health care.
Explaining male mortality differentials between the west of Scotland and the south of England

GEORGE DAVEY SMITH, ${ }^{1}$ MARTIN SHIPLEY, ${ }^{2}$ DAVID HOLE, ${ }^{3}$ CAROLE HART, ${ }^{3}$ GRAHAM WATT, ${ }^{3}$ CHARLES GILLIS, ${ }^{3}$ M G MARMOT, ${ }^{4}$ VICTOR HAWTHORNE ${ }^{3}{ }^{1}$ Department of Social Medicine, University of Bristol; ${ }^{2}$ Department of Epidemiology and Public Health, University College London; ${ }^{3}$ Departments of Public Health and General Practice, University of Glasgow; ${ }^{4}$ Department of Epidemiology and Population Sciences, London School of Hygiene and Tropical Medicine) Background - Mortality differences from various causes exist between Scotland and England. Cohort studies in which mortality can be related to individual risk factors allow for detailed examination of this issue. One cohort in London and two cohorts in the west of Scotland were established around the same time and utilised similar screening procedures in similar age groups. The mortality experiences of these three cohorts have therefore been compared.

Design - Prospective cohort studies.

Subjects and setting - The Whitehall study of London based civil servants included 15637 men aged 45-64 years screened between 1967 and 1970; the collaborative study of employees in a wide variety of workplaces around Glasgow included 3825 men aged 45-64 years screened between 1970 and 1973; the Renfrew/Paisley study, a representative community based survey, included 7058 men aged 45-64 years screened between 1972 and 1976.

Main outcome measures - Mortality from a variety of causes.

Results - The distribution of poor health at baseline was least favourable in the Renfrew/ Paisley study and most favourable in the Whitehall study. In the first 10 years of follow up, mortality rates were much higher in the Renfrew/Paisley study than in either the collaborative or Whitehall studies. After the initial 10 years, both the collaborative and the Renfrew/Paisley studies had higher mortality than the Whitehall study. This was true for a variety of causes of death. The relative rates of mortality between the west of Scotland cohorts and the Whitehall study were computed with adjustments for age, height, $\mathrm{FEV}_{1}$, socioeconomic status, and smoking. The raised mortality in these cohorts was substantially attenuated by such adjustments, indicating that differences in a few basic characteristics account for much if not all of the mortality differentials between the south of England and the west of Scotland.

Conclusions - Differences in mortality between the west of Scotland cohorts and the Whitehall study are not explained by factors which are particular to the west of Scotland. Rather they are explained by factors which are comrion to both populations, which are more prevalent in the west of Scotland.

Are towns in England and Wales less healthy?

JOHN CHARLTON (Office of Population Censuses and Surveys, London)

Objectives - To compare health status by geography over time (1981-92), and to update William Farr's 1841 life tables for Liverpool, London, and Surrey.

Materials and methods - Mortality data by local authority (1981-92) and ward (1990-92) Prevalence data by enumeration district (ED) 
from the 4th national GP morbidity survey (1991-92). Comparisons in terms of life expectancy, age standardised mortality for all causes and selected key causes, and disease prevalence as recorded by GPs. The OPCS area classification based on 1991 census characteristics groups the 403 local authorities into 11 groups, including "Rural", "Inner London", "Ports and Industry" (e.g. Liverpool), and "Most prosperous" (most of Surrey). The Department of Environment's urban/rural classification of EDs was used to classify wards into "rural", "urban", and "mixed".

Results - In 1990-92, urban areas were still less healthy than rural areas on most indicators - they had higher mortality and higher GP consultation rates for serious illness. Since 1981, the "Most prosperous" areas have shown the greatest health gains, particularly for men, where life expectancy at birth increased by $4.5 \%$ to 76 years. "Rural" areas improved almost as much, to 75 years. "Inner London" and "Ports and Industry" have made the smallest health gains, to 72 years. In 1992 the league table published by Farr in 1841 still stands. In contrast, however, the worst life expectancies for women in 1992 were better than the best for men, since women's health gains have by far outstripped those for men. Out of every 100000 boys born in "Ports and Industry" areas, 16000 would survive to age 85 years, whereas 24000 would do so in the "Most prosperous" areas. The corresponding figures for girls were 33000 and 43000 . The "Most prosperous" areas also had the most equal male and female life expectancies, equality improving throughout the period. In "Inner London" there was no catching up. Turning to causes of death, "Ports and Industry" had the highest male SMRs in 1990-92 for malignant neoplasms, lung cancer, circulatory diseases, ischaemic heart disease, and cerebrovascular disease. "Inner London" had the highest SMRs for respiratory diseases and injury and poisoning. Conclusion - Urban areas are less healthy then rural areas. The healthiest areas are those in the "Most prosperous" category. The least healthy are "Inner London" and "Ports and Industry", where there has been least health gain since 1981. Health status varies more among males than females, and during 1981-92 male life expectancy has caught up most with female life expectancy in the "Most prosperous" areas.

METHODOLOGY

Evidence for the sensitivity of the short form-36 health status measure to inequalities in health

CRISPIN JENKINSON, RICHARD LAYTE (Health Services Research Unit, Department of Public Health and Primary Care, University of Oxford) Objectives - A recent paper suggested that the short form-36 (SF-36) may not be appropriate for population surveys assessing health gain because of a lack of sensitivity of the eight dimensions of the SF-36 to social class inequalities in health. In this paper, however, we show that the SF-36 is sensitive to social class differences, and indeed, with further analysis, can show dramatic health status contrasts between the worst and best off in society.

Design - This was a postal survey using a questionnaire booklet containing the SF-36 and a number of other items concerned with lifestyles and illness. A letter outlining the purpose of the study was included. Setting - The sample was drawn from family health services authority (FHSA) computerised registers for Berkshire, Bucking hamshire, Northamptonshire, and Oxfordshire.

Sample - The questionnaire booklet was sen to 13042 randomly selected subjects aged 17-65 years. This paper is based upon the $9332(72 \%)$ responses gained.

Outcome measures - Scores for the eight dimensions of the SF-36.

Statistics - The sensitivity of the SF-36 was tested by hypothesising that the scores of those in the bottom quartile of the SF-36 scores in social class $\mathrm{V}$ could be improved to the level of the scores from the bottom quartile of SF-36 scores in class I using the effect size statistic.

Results - The response rate for the questionnaire booklet was $72 \%$. Completion of all items in individual scales was high, although over $12 \%$ of respondents omitted at least one question from the measure. We provide SF-36 scores for the population at the 25 th, 50th, and 75 th centiles. Those reporting worse health on each dimension of the SF36 (that is, in the lowest $25 \%$ of scores) differ dramatically between social class I and V. Large effect sizes were gained on all but one dimension of the SF-36 when the scores of those in the bottom quartile of the SF-36 scores in class $\mathrm{V}$ were hypothesised to have improved to the level of the scores from the bottom quartile of SF-36 scores in class I.

Conclusions - The SF-36 is sensitive to population variations in health status. Analysing the data as a whole provides an inaccurate picture of the level of ill health in the worst of in society. Those reporting the worst health in class $\mathrm{V}$ report far worse functioning and well being than those reporting the worse health in class I. Not only are differences between the classes detected by the SF-36 but they are more dramatic than might previously have been imagined.

Use of the short form 36 general health survey in an occupational cohort: age, sex and socioeconomic status differences

HARRY HEMINGWAY, AMANDA NICHOLSON, RON ROBERTS, MAI NGUYEN, MICHAEL MARMOT ( $D e-$ partment of Epidemiology and Public Health, University College London Medical School Background - The short form-36 (SF-36) is increasingly being used as a measure of the effectiveness of medical care. There are few reports of association between the SF-36 and socioeconomic status.

Objective - To describe age, sex, and socioeconomic status differences in scale scores of the SF-36 general health survey in the Whitehall II study.

Design - Questionnaire of study participants. Setting - Twenty civil service departments originally located in London.

Subjects - Altogether 5929 male and 2683 female civil servants aged 39 to 63 years.

Main outcome measures - The eight scales within the SF-36 profile: physical functioning, role limitations due to physical functioning, bodily pain, general health perceptions, vitality, social functioning, role limitations due to emotional problems, and general mental health.

Results - There were significant $(\mathrm{p}<0.0001)$ improvements with age in general mental health, role-emotional problems, vitality, and social functioning scale scores in men and women, with average increase in scale score associated with each year increase in age between 0.09 and 0.41 . Physical functioning scores deteriorated $(\mathrm{p}<0.0001)$ with age (regression coefficient -0.36 in men and -0.59 in women). For each scale except general health perceptions, women scored consistently lower than men at every age group $(\mathrm{p}<0.05)$ and employment grade. Among men there were significant $(p \leq 0.0002)$ age adjusted gradients across the six civil service employment grades in all the scales except vitality, such that the higher status grades reported better health. Among women a similar relation was found for the physical functioning, pain, and social functioning scales. Age and grade adjusted scores on each scale were lower among women without access to a car ( $\mathrm{p} \leq 0.03)$.

Conclusions - Low socioeconomic status was associated with low scale scores; the effect was comparable in magnitude to that of some clinical conditions. Socioeconomic status factors should be considered in the interpretation of SF-36 data. Decline in health functioning with age should not be assumed.

\section{The assessment of "avoidable" death}

J TURNER, J P NICHOLL (Medical Care Research Unit, University of Sheffield)

Purpose - To develop a comprehensive method for the assessment of "avoidable" death as an outcome measure of trauma service performance.

Methods - Case profiles containing details of pre-hospital events, accident and emergency department treatment, injury descriptions, and a summary of inpatient events were constructed for 400 deaths entered into an evaluation of a regional trauma system. Assessment of avoidable death was made by a five member expert panel. The assessment process comprised three stages: (1) An assessment of whether the injuries alone were survivable. (2) If yes, a judgement about whether they were survivable for the individual patient taking into account the circumstances of the incident and relevant patient characteristics, and (3) if yes, an assessment of the most appropriate level of care required and a score on a scale 1-5 indicating the probability that the outcome could have been reversed. Errors of omission and commission which contributed to avoidable death were also identified. Cases were initially reviewed individually, and assessed for consensus. Consensus required was $5: 0$ or $4: 1$. Equivocal cases were discussed at periodic meetings and consensus obtained.

Results - The 400 reviewed cases will be presented. Changes in the avoidable death rate in a regional trauma system and two comparator regions over a four year period will be discussed. Analysis of the most appropriate levels of care, the scoring method for assessing certainty, and the management errors identified will also be presented and discussed.

Conclusions - This method provides several improvements on previous attempts to assess avoidable death. Explicit distinction is made between survivable injury and avoidable death, and factors other than injury, for example incident circumstances and co-morbidities, can be taken into account in the assessment process. When death is judged to be avoidable consideration is given to the 
different levels of care appropriate to different injuries and a systematic appraisal of factors contributing to avoidable death can be made.

\section{Investigation of heterogeneity among trials in meta-analysis: a preliminary review}

F SONG, T A SHELDON (NHS Centre for Reviews and Dissemination, University of York)

Objectives - To conduct a preliminary review of sources of heterogeneity and methods of its investigation in meta-analyses.

Design - Abstracts of published meta-analyses, located by searching Medline (1994), were scanned to identify those that considered heterogeneity among primary studies. Selective meta-analyses were examined in depth as examples to explore sources of heterogeneity and methods of investigation.

Results - A limited Medline search listed 165 abstracts of meta-analyses. Sixty eight were excluded for reasons such as being an epidemiological study, a guideline, a case study. Fifty one of 97 remaining studies (53\%) discussed heterogeneity (or homogeneity) in the abstract. The most common source of heterogeneity was variation in patient characteristics (for example, criteria for inclusion, severity of illness, and level of baseline risk). Other sources of heterogeneity included variation in the interventions (for example, concomitant treatment, dosage, control interventions), variation in outcome measures, publication bias (for example, treatment effect related to sample size), and methodological quality of primary trials. In addition to presenting data from individual studies in figures and tables, the statistical test of homogeneity, subgroup analysis, and regression analysis (linear and logistic) were common methods used to investigate heterogeneity. The investigation of heterogeneity in a meta-analysis could produce suggestive information on factors that influence effectiveness of a health intervention.

Conclusions - An overall or average estimate of treatment effect may be misleading if considerable heterogeneity exists among primary trials. If currently available methods were used appropriately, investigation of heterogeneity could be useful to explore data from primary studies more thoroughly, to identify patient groups who might benefit most from a health intervention, to understand how a health intervention should be provided, to improve quality of primary trials, and to indicate the direction of future research.

\section{ETHNICITY}

Racial discrimination in medicine: a blot on the profession

A ESMAIL (Department of General Practice, University of Manchester)

Background - Several studies have been carried out over the past two years showing how racial discrimination affects entry to medical school, job applications for junior hospital posts, and disciplinary procedures against doctors. Together they provide the most comprehensive assessment of the barriers faced by ethnic minorities in the pursuit of a career in medicine. White applicants to medical school are between two and 10 times more likely to be accepted for a place compared with ethnic minority applicants despite having similar A-level results. White doctors are twice as likely to be shortlisted for a junior hospital post compared with ethnic minority doctors with the same qualifications. However, ethnic minority doctors are six times more likely than their white colleagues to be brought before the professional conduct committee of the General Medical Council. Although the individual studies received widespread coverage in the medical and lay press, the failure of the bodies representing the medical profession (including the General Medical Council) to define a comprehensive strategy to tackle the problem of discrimination is a glaring omission.

This paper will review current evidence on the extent of discrimination in the medical profession, describe the response of the medical profession to the evidence, discuss the responsibilities of researchers in highlighting the problem and suggest a strategy on how it should be tackled.

Ethnicity and mental health: findings from a recent census of acute psychiatric beds in North and South Thames Health Regions

J S KOFFMAN, N. J. FULOP (Kensington and Chelse and Westminster Health Commissioning Agency, London)

Objective - To investigate the proportion and characteristics of different ethnic groups occupying adult acute and low level secure psychiatric beds.

Design - Point prevalence survey of adult acute and low level secure psychiatric inpatients.

Setting - All NHS adult acute and low level secure psychiatric units and seven private psychiatric units in North and South Thames Health Regions.

Subjects - A total of 3710 adult acute and 268 low level secure psychiatric inpatients on 15 June 1994.

Main outcome measures - Sociodemographic, admission, and clinical characteristics of patients.

Results - Compared with the 1991 census population of North and South Thames Health Regions, the black population were over represented in this psychiatric inpatient population $\left(15.8 \% v 5 \cdot 2 \%, \chi^{2}=1922.67\right.$, $\mathrm{p}<0.001)$. Asian patients were under represented $(4.0 \% v 4.6 \%)$ as were white patients $(74.8 \% v 87 \cdot 7 \%)$ compared with the overall population. Altogether $63.5 \%(n=399)$ of black patients compared with $38.8 \%$ ( $n=$ $62)$ of Asian and $30.6 \%(n=907)$ of white patients were admitted under the provisions of the Mental Health Act (1983) $\left(\chi^{2}=257\right.$, $\mathrm{p}<0.001)$. Furthermore, $10.4 \%(n=66)$ of black patients were located in locked wards compared with $3 \cdot 1 \%(n=5)$ of Asian and $6 \cdot 1 \%(n=182)$ of white patients $\left(\chi^{2}=18 \cdot 10\right.$, $p=0.0004)$. A total of $25.3 \%(n=157)$ of black patients had been admitted on two or more occasions in the previous 12 months to psychiatric units compared with $22.0 \%$ ( $n=$ 34) of Asian and $23 \cdot 3 \%(n=675)$ of white patients $\left(\chi^{2}=14.68, p=0.25\right)$. High rates of the diagnosis of schizophrenia were not confined to black patients $(71 \cdot 1 \% \quad n=444)$ $51 \cdot 2 \%(n=82)$ of Asian patients were diagnosed with schizophrenia compared with $36.0 \%(n=1062)$ of white patients $\left(\chi^{2}=\right.$ $347 \cdot 95, \mathrm{p}<0 \cdot 001)$.
Conclusions - These results support evidence that the black population in Britain have high admission rates to inpatient psychiatric services, are frequently admitted formally, and are over represented in secure units. High rates of the diagnosis of schizophrenia are not confined to the black ethnic minority population; over half of Asian psychiatric patients have a diagnosis of schizophrenia. Another recent study also found high rates of psychosis in Asians. This study's findings will be discussed in relation to: the perception that mental illness among black patients is more "antisocial" than in other ethnic groups; alternatives to hospital admission are less likely to be offered to black patients; and the personal and social pressures of belonging to an ethnic group lead to higher rates of diagnosed schizophrenia.

Does ethnicity influence the patterns of asthma treatment in children?

E DURAN-TAULERIA, P BURNEY, R J RONA, S CHINN (Department of Public Health Medicine, UMDS, St Thomas's Campus, London)

Background - Socioeconomic and ethnic differences in asthma drug management have been shown in New Zealand and the USA. Although several studies have investigated problems of undertreatment in the UK, the relationship between ethnic origin and treatment has not been studied in population based studies.

Objective - To explore the influence of ethnicity and sociodemographic factors on asthma drug treatment in children.

Design - The study has a cross sectional design. The data were obtained from the national study of health and growth, a population based survey of primary school children living in England and Scotland. Information was obtained from a self administered questionnaire, usually completed by the child's mother. The study included a representative sample of 6463 children living in England in 1990 and 4165 in Scotland in 1990 or 1991 . The inner city English 1991 sample included 7049 children -2183 white children, and 4866 from ethnic minority groups.

Results - The response rate was $92.3 \%$ for the representative sample and $85.3 \%$ for the inner city sample. Respiratory symptoms were reported for $5483(31 \%)$ children. Of those reporting wheezing sometimes and having had asthma attacks (877), only $79 \%$ had been prescribed $\beta_{2}$-adrenergic drugs and only $65 \%$ of these were taking inhaled rather than oral medication. Those children living in a inner city area were less likely to have the drugs prescribed compared with those in the English representative sample (odds ratio 0.50 $(95 \%$ CI $0.33,0.75)$. The odds ratios for ethnic groups were $0.36(95 \% \mathrm{CI} 0.18,0.71)$ for Afro-Caribbean children, and within the Indian groups the odds ratio ranged from 0.29 to 0.41 compared with the 1990 English group. Social class was associated with treatment $(p=0.008)$. Children in social class IIIM were less likely to be prescribed $\beta_{2}$ adrenergic drugs (odds ratio 0.48 (95\% CI $0.28,0.84$ ) than those in the social class I and II group. Similar associations to that described for $\beta_{2}$-adrenergic drugs were found for prophylactic drugs.

Conclusion - Ethnicity and social class were associated with prescribed drugs for asthma treatment. Children living in inner city areas 
are less likely to have appropriate treatment. Programmes to improve the effectiveness of asthma treatment in children need to take these factors into account.

CARDIOVASCULAR EPIDEMIOLOGY (I)

Size at birth and blood pressure and fasting glucose at age 50 - how important is confounding by sociodemographic and behavioural factors?

I KOUPILOVÁ, ${ }^{12}$ D A LEON ${ }^{1}$ D VÅGERÖ, ${ }^{3}$ U-B Lithell, ${ }^{2}$ P MCKEIGUE, ${ }^{1}$ H LITHELL ${ }^{2}$ ( ${ }^{1} E p i-$ demiology Unit, London School of Hygiene and Tropical Medicine; ' Institute of Geriatrics, University of Uppsala; ${ }^{3}$ Swedish Institute for Social Research, University of Stockholm)

Objective - To evaluate whether socioeconomic confounding explains the relationship between size at birth and blood pressure and fasting glucose at the age of 50 years.

Design - Cross sectional study with retrospectively collected data on size at birth Setting - Uppsala, Sweden.

Subjects - A total of 924 men born 1920-24. Main outcome measures - Blood pressure after 10 minutes rest and fasting blood glucose concentration were measured. Data on parental profession and marital status were taken from health records. Data on the subjects' own sociodemographic and behavioural characteristics were taken from self completed questionnaires. The results are adjusted for body mass index at age 50

Results - There was a strong negative association between birth weight and systolic blood pressure at the age of $50(4.58,6.08$ and $1.49 \mathrm{~mm} \mathrm{Hg}$ excess in birth weight group $<3250$ g, 3250-, 3750- compared with $4250+\mathrm{g}$, $\mathrm{p}$ value for trend $0 \cdot 003$ ). This was strengthened by adjusting for the subjects' highest education level and adult height, while adjustment for current alcohol drinking habits slightly reduced the strength of the association. The subjects' own marital status, social class, and area of birth did not confound this relationship. Simultaneous adjustmen for drinking habits and all the socioeconomic indicators strengthened the negative association between birth weight and blood pressure $(5 \cdot 64,6 \cdot 46$, and $1.83 \mathrm{~mm} \mathrm{Hg}$ exces in the respective birth weight groups, $\mathrm{p}$ value for trend $<0 \cdot 001$ ). In 470 study subjects, data on parents' marital status and social clas were available. Adjustment for these factor strengthened the association between birth weight and blood pressure (excess in the re spective birth weight groups $5.60,5 \cdot 13$, $1.52 \mathrm{~mm} \mathrm{Hg}$ before, and 5.79, 5.44, $1.61 \mathrm{~mm}$ $\mathrm{Hg}$ after this adjustment, $\mathrm{p}$ values for trend 0.051 and 0.048 ). None of the socioeconomic indicators were significantly related to the ponderal index (weight/length ${ }^{3}$ ) at birth, and adjustment for them did not significantly affect the negative association between the ponderal index at birth and the fasting glucose value at age $50(0 \cdot 24,0 \cdot 05,0 \cdot 04,0.06 \mathrm{mmol} /$ 1 excess in the lowest four ponderal index quintiles, $\mathrm{p}$ value for trend 0.036 )

Conclusion - None of the socioeconomic in dicators could explain the association between birth weight and adult blood pressure. As none of the socioeconomic indicators are related to ponderal index at birth, the as sociations of ponderal index at birth with adult metabolic characteristics are unlikely to be due to socioeconomic confounding.
Influence of birth weight and maternal smoking during pregnancy on blood pressure in children

S TAYLOR, ${ }^{1}$ P H WHINCUP, ${ }^{1}$ D G COOK, ${ }^{2}$ O PAPACOSTA, ${ }^{1} \mathrm{M}$ WALKER, ${ }^{1} \mathrm{~V}$ WILSON ${ }^{1}\left({ }^{1}\right.$ Department of Public Health, Royal Free Hospital School of Medicine, London; ${ }^{2}$ Department of Public Health Sciences, St George's Hospital Medical School, London)

Background - Low birth weight has been linked to higher blood pressure, and the relationship has been reported to become stronger ("amplified") with increasing age. However, it has recently been suggested that maternal smoking during pregnancy is related to lower subsequent blood pressure in early, preterm infants.

Objective - To determine whether birth weigh and maternal smoking in pregnancy are related to childhood blood pressure.

Design and subjects - This was a school based survey of children aged 9-11 years, linked to earlier assessment at 5-7 years in a subgroup. Measurements - Duplicate measurements of sitting blood pressure, height and weight were made; birth weight, length of gestation, and aternal smoking during pregnancy were assessed by maternal recall.

Results - A total of 3728 children attended for physical measurements $(75 \%$ response rate); questionnaire data were available in 3072 children (response rate $62 \%$ ). Birth weight showed an inverse relationship with blood pressure once height and the ponderal index were taken into account (systolic blood pressure $=-1.60 \mathrm{mmHg} / \mathrm{kg}, 95 \% \mathrm{CI}$ $-0.90,-2.30 \mathrm{mmHg} / \mathrm{kg}$ ). However, in 1961 children with longitudinal measurements, the relationship seemed to be becoming weaker rather than stronger than that observed at age 5-7 years, with the change in blood pressure between 5-7 and 9-11 years showing a weak positive relation with birth weight (systolic pressure mean $(\mathrm{SEM})=0.21 \mathrm{~mm}(0.46) \mathrm{Hg}$ $\mathrm{kg})$. Maternal smoking during pregnancy was associated with a reduction in the mean birth weight of $0.20 \mathrm{~kg}(95 \%$ CI $0.16,0.24 \mathrm{~kg})$ However, no overall relationship between maternal smoking and blood pressure at 9-11 years was observed either in univariate analysis or after adjustment for height, pondera index, and birth weight. In 40 children born before 34 weeks of gestation $(1.3 \%$ of the study population), the mean (SEM) systolic pressure was $2.7(2.3) \mathrm{mmHg}$ lower in children whose mothers had smoked during pregnancy. However, this difference was not statistically significant and no formal evidence of interaction between maternal smoking and gestational age was observed.

Conclusions - The results confirm earlier reports of an inverse association between birth weight and blood pressure but do not support the possibility of amplification during the later part of the first decade. At the population level, maternal cigarette smoking during pregnancy does not seem to have an important influence on blood pressure in childhood.

\section{Cardiovascular risk factors in British children in towns with widely differing adult cardiovascular mortality rates}

P H WHINCUP, ${ }^{1}$ D G COOK, ${ }^{2}$ F ADSHEAD, ${ }^{2} \mathrm{~S}$ TAYLOR, ${ }^{1}$ O PAPACOSTA, ${ }^{1}$ M WALKER, ${ }^{1} \mathrm{~V}$ WILSON ${ }^{1}$ ('Department of Public Health, Royal Free Hospital School of Medicine, London; ${ }^{2}$ Department of Public Health Sciences, St George's Hospital Medical School, London)
Background - The "Barker hypothesis" claims that early life factors play an important part in explaining both individual and geographic variations in cardiovascular risk.

Objective - This study aimed to examine whether cardiovascular risk factors implicated in this hypothesis (particularly blood pressure, total cholesterol, waist-hip ratio, and 30 minute post-load glucose) differ in children from towns with widely differing adult cardiovascular mortality.

Design - This was a school based survey in five towns with exceptionally low adult cardiovascular mortality (SMR 64-75) and five with exceptionally high adult cardiovascular mortality (SMR 131-143) conducted during 1994. Towns were surveyed in high-low pairs

Subjects - Altogether 3406 children of European origin aged 9-11 years had physical measurements taken (response rate $75 \%$ ), and 1277 provided blood samples (response rate $64 \%$ ).

Results - Children in high mortality towns were on average $1.2 \mathrm{~cm}$ shorter than those in low mortality towns $(95 \%$ CI $0.3,2 \cdot 1 \mathrm{~cm}$, $\mathrm{p}=0.02$ ). Mean blood pressures were slightly higher in high mortality towns, but only when adjusted for current differences in height (mean systolic difference $2.0 \mathrm{mmHg}, 95 \% \mathrm{CI}$ $0.8,3.2 \mathrm{mmHg}, \mathrm{p}=0.02)$. There were no important differences between high and low mortality towns in waist-hip ratio, 30 minute post-load glucose measurements, or in total or low density lipoprotein cholesterol concentrations

Conclusions - The hypothesis that early life factors are important determinants of geographic variation in cardiovascular mortality in Britain is based on the study of subjects born in the early years of the 20th century. The absence of consistent risk factor differences in contemporary children between these high and low mortality towns (which differ by $75 \%$ in their average perinatal mortality rate and by $30 \%$ in their average prevalence of low birth weight) suggests that the "Barker hypothesis" will have limited relevance to geographic variation in cardiovascular disease in this generation.

\section{Does socioeconomic status in childhood} affect cardiovascular risk in adulthood?

G WANNAMETHEE, P H WHINCUP, A G SHAPER, M WALKer (Department of Public Health, Royal Free Hospital School of Medicine, London)

Objective - To examine the relation between socioeconomic status in childhood and cardiovascular risk factors and prevalent myocardial infarction in adulthood.

Design - This was a longitudinal study of cardiovascular disease, with cross sectional measurement survey in 1978-80 and follow up questionnaire survey in 1992 (seeking information on childhood socioeconomic status and prevalent disease)

Subjects - These comprised 5934 men aged 40-59 years at entry to the study who completed the 1992 follow up questionnaire (77\% of original participants).

Socioeconomic variables and outcome measures

- Two measures of childhood socioeconomic status were assessed from the 1992 questionnaire: (i) father's occupation (manual/ non-manual) and (ii) family amenity score up to age 10 , based on car ownership, domestic bathroom, and hot water provision. Adult social class was based on the longest held occupation at study entry. Outcomes in- 
cluded (i) cardiovascular risk factors at screening and (ii) prevalence of myocardial infarction at 1992 questionnaire survey.

Results - Both measures of socioeconomic status in childhood ( $\mathrm{i}$ and ii) were strongly associated with social class in adulthood. Low socioeconomic status in childhood (i and ii) was significantly related to shorter stature and lower lung function $\left(\mathrm{FEV}_{1}\right)$ even after adjustment for adult social class. Men from a manual working background tended to be more obese than those from a non-manual background, irrespective of adult social class. While no association was seen with blood glucose, both measures of childhood socioeconomic status were related to systolic blood pressure, blood cholesterol, high density lipoprotein cholesterol, smoking, and physical activity but these relationships were attenuated after adjustment for adult social class. Family amenity score showed no association with prevalence of myocardial infarction. However, men from a manual working background had a significantly higher prevalence of myocardial infarction $(\mathrm{RR}=1.4 ; 95 \%$ CI $1 \cdot 2,1 \cdot 6)$ compared with men from a non-manual childhood background, even after allowing for adult social class and other established risk factors.

Conclusion - Childhood socioeconomic status is strongly associated with adult social class. Several associations between socioeconomic status in childhood and cardiovascular risk factors in adulthood are present but these are confounded by adult social class. Despite this, the finding that men from manual working background showed a higher prevalence of myocardial infarction independent of adult social class or risk factors suggests that childhood socioeconomic status may have some lasting influence on cardiovascular risk in adult life.

Past or present Helicobacter pylori infection and fibrinogen - a possible link between social class and coronary risk?

ERIC BRUNNER, ${ }^{1}$ MICHAEL MENDALL, ${ }^{2}$ MICHAEL MARMOT ${ }^{1}$ ( ${ }^{1}$ Department of Epidemiology and Public Health, University College London: ${ }^{2} \mathrm{De}-$ partment of Medicine, St George's Hospital Medical School, London)

Objective - It has been suggested that persistent infection may increase risk of coronary heart disease (CHD), the effect being mediated by the long term low grade acute phase response to that infection. In support of this, a case-control study showed $H$ pylori infection was linked to non-fatal CHD, and further that seropositives among controls had a mean fibrinogen $0.43 \mathrm{~g} / 1(0.67 \times \mathrm{SD})$ higher than seronegatives after adjustment for smoking, father's social class (Registrar General), own social class, and childhood crowding. Since $H$ pylori infection is associated with poor childhood living conditions it may be speculated that lifelong infection could be a partial explanation for the link between low socioeconomic status and CHD. To examine this proposition we conducted an investigation with two primary objectives: to confirm the inverse social gradient in $H$ pylori seropositivity and to examine its association with plasma fibrinogen levels and other coronary risk factors.

Design - This was a cross sectional study. Setting - London based civil servants. Subjects - Stratified random subsample of 347 men and women aged 45-55 years from the
Whitehall II study baseline selected on the basis of sex, age, and employment grade. Serum stored at $-70^{\circ} \mathrm{C}$ was analysed for $H$ pylori specific IgG antibodies.

Results - Some $30 \cdot 7 \%$ (base $=186$ ) of men and $28.6 \%$ (base $=161$ ) of women were classified seropositive. The age adjusted prevalences of $H$ pylori seropositivity according to civil service employment grade among men are: clerical $51.8 \%$, executive $24.3 \%$, and administrative $31.5 \%$ (trend $\mathrm{p}=0.37$ ), and among women: clerical $41.7 \%$, executive $26.0 \%$, and administrative $23.5 \%$ (trend $\mathrm{p}=$ 0.07 ). Analysis of the associations of $H$ pylor seropositivity with fibrinogen, white blood cell count, factor VII, serum cholesterol, and apolipoproteins AI and B shows that none were statistically significant. Measures of the acute phase response - plasma fibrinogen and white blood cell counts - show the expected tendency in three out of four contrasts. Seropositives, especially women, tended to have a poorer risk profile; however associations were frequently weakened by adjustment for employment grade.

Conclusion - We have shown that past or present $H$ pylori infection is weakly linked with lower socioeconomic status. Our findings do not support the view that $H$ pylori may mediate the link betwen low socioeconomic status and CHD risk, since seropositivity is not specifically related to raised fibrinogen, white blood cell count, or other risk factor levels in this study. It may be that $H$ pylori seropositivity is not causally related to adult CHD but is a marker of childhood deprivation, which is itself implicated in later risk of $\mathrm{CHD}$ through several putative mechanisms.

Long term vascular sequelae of toxaemia of pregnancy

$S$ FERRY, S HIRSCH, P HANNAFORD (Royal College of General Practitioners' Manchester Research Unit)

Background - Several studies have shown that women with a history of toxaemia of pregnancy subsequently have an increased risk of hypertension. Comparatively little is known about other vascular sequelae.

Objectives - To determine whether toxaemia of pregnancy is associated with an increased risk of various vascular diseases in later life. Design - Since the late 1960s, the Royal College of General Practitioners' oral contraception study has followed an initial cohort of 23000 pill users and 23000 non-users in order to determine the effects of the contraceptive pill. Parous women in the study who had never used oral contraceptives were categorised according to whether or not they had a history of toxaemia of pregnancy documented. The rates of first ever hypertension, myocardial infarction, angina, total ischaemic heart disease, total stroke, and peripheral vascular disease were calculated for each group, after direct standardisation for smoking, social class, age, and the number of pregnancies. By September 1993, the women with toxaemia of pregnancy had experienced 488 events during 30957 women years of observation; those without such a history had experienced 1317 events during 161963 women years.

Results - Compared with women without a history of toxaemia of pregnancy, those with such a history had a significantly increased risk of hypertension (relative risk (RR) $2 \cdot 02$, $95 \%$ confidence interval (CI) $1 \cdot 78,2 \cdot 29$ ), myocardial infarction (RR $2 \cdot 24,95 \% \mathrm{CI} 1 \cdot 42$, 3.52 ), angina pectoris ( $R R 1.60,95 \% \mathrm{CI}$ $1 \cdot 15,2.23)$ and total ischaemic heart disease (RR $1.73,95 \%$ CI $1.32,2 \cdot 26$ ). The risk of stroke was also slightly raised but did not reach statistical significance, possibly because of the small number involved (RR 1.24, 95\% CI $0.77,2 \cdot 00$ ). Only 13 women with a history of toxaemia of pregnancy and 53 women without such a history had a subsequent diagnosis of peripheral vascular disease; too few for analysis.

Conclusion - A history of toxaemia of pregnancy seems to be associated with hypertension, ischaemic heart disease, and possibly stroke in later life. In this study, however, predefined criteria for the diagnosis of toaxemia of pregnancy were not available. The diagnosis is likely to include a variety of problems of blood pressure in pregnancy. Other prospective studies which have a more stringent definition of toxaemia of pregnancy are needed in order to substantiate our findings.

Aspirin in the prevention of cardiovascular disease: are opportunities being missed?

A $\mathrm{K}$ MCCALLUM, P H WHINCUP, R W MORRIS, M WALKER, S EBRAHIM (Department of Public Health, Royal Free Hospital School of Medicine, London)

Background - The benefits of aspirin in the secondary prevention of coronary heart disease have been supported by clinical tria evidence since the 1980 s. Although hospital based studies of aspirin use have been conducted, little is known about the use of aspirin by patients with cardiovascular disease in the general population

Objective - To explore use of aspirin by patients with cardiovascular disease.

Design - Longitudinal study of cardiovascular disease (British regional heart study), in which subjects have been followed up for cardiovascular morbidity and mortality since entry in 1978-1980. Data on cardiovascular diagnoses in this report are based on general practice notifications up to September 1992.

Data on daily aspirin use were based on a postal questionnaire to survey participants despatched between October and December 1992 (Q92).

Subjects - Analyses are based on 5934 men aged 52-74 years who completed Q92. This constitutes $90 \%$ of the survivors of the original cohort of 7735 men. Detailed information on aspirin use was available for 5751 men $(87 \%$ of survivors).

Results - Overall, 547 men $(9 \cdot 2 \%$ of the study population) were taking daily aspirin. Of these subjects, more than half $(52 \%)$ had a previous diagnosis of coronary heart disease or stroke. Among subjects with previous myocardial infarction $(n=342), 148(43 \%)$ were taking aspirin daily, as were $35 \%$ of those with previous stroke $(n=92)$. A lower proportion of those with a history of angina $(n=255)$ was receiving daily aspirin (28\%). Of those with previous myocardial infarction who had also had a coronary angioplasty or a coronary artery bypass graft, daily aspirin use was reported by $24 / 41$ patients (59\%) compared with $124 / 301(41 \%)$ in those who had not $(\mathrm{p}=0.05)$.

Conclusions - Despite strong evidence of effectiveness, the results suggest that at the time of this survey many patients with es- 
tablished cardiovascular disease were not receiving aspirin, particularly those who had not undergone invasive treatment. One simple method of reducing mortality from cardiovascular disease is not therefore being fully exploited. Further valuable information about possible barriers to aspirin use would be obtained from community based collaborative audit of treatment, particularly examining the roles of those involved in primary and secondary care cardiac and stroke rehabilitation programmes.

GENERAL PRACTICE (II)

Consulting the general practitioner for symptoms of minor illness: why are women more likely to consult?

SALLY WYKE, ${ }^{1}$ KATE HUNT, ${ }^{2}$ GRAEME FORD $^{2}$ ${ }^{1}$ Department of General Practice, University of Edinburgh; ${ }^{2} M R C$ Medical Sociology Unit, Glasgow)

Objective - To investigate gender differences in consulting behaviour in response to symptoms of minor illness and possible explanations for these differences.

Design - Analysis of consulting behaviour in response to symptoms in relation to: gender number of other symptoms experienced and number restricting activity (number reported either out of a total of 33 symptoms of minor illness; a total of seven symptoms of malaise; a total of nine physical symptoms); number of other long standing illnesses; number of dependent children; number of hours in paid work; age; social class; marital status; and self reported employment status.

Main outcome measures - Consultation reported to nurse interviewers for any of: total number of symptoms reported; number of symptoms of malaise; number of physical symptoms.

Respondents - Respondents in two age cohorts (852 aged 39 and 858 aged 58) who took part in the west of Scotland twenty-07 study in 1991 .

Results - Women were more likely to have consulted their general practitioner for at least one of the total number of symptoms they reported $\left(58 \%\right.$ of women, $42 \%$ of men; $\chi^{2}=$ $6 \cdot 8, \mathrm{p}<0.01$ ); this was true for consulting for symptoms of malaise $(65 \%$ of women, $35 \%$ of men, $\left.\chi^{2}=10.9, \mathrm{p}<0.001\right)$; and for consulting for physical symptoms $(63 \%$ of women, $37 \%$ of men, $\left.\chi^{2}=12 \cdot 0, p<0.001\right)$. In multivariate analysis, gender did not contribute to the prediction of likelihood of consultation either for total number of symptoms reported (predictors were: symptoms reported $(\mathrm{OR}=1 \cdot 19,95 \% \mathrm{CI}=1 \cdot 14,1 \cdot 25)$; symptoms that restricted activity $(1 \cdot 28,1 \cdot 18$, 1.39); age cohort $(1.6,1.28,2.01)$; long standing illnesses $(1.22,1.07,1.37)$; being unemployed $(1.70,1.06,2.72))$, or for symptoms of malaise reported (predictors were: symptoms of malaise reported (1.8, $1.60,2.03)$; symptoms of malaise that restricted activity $(1 \cdot 76,1 \cdot 44,2 \cdot 16)$; long standing illness $(1 \cdot 23,1 \cdot 04,1 \cdot 45)$; hours per week in paid work $(0.99,0.98,1.00))$. However, gender did contribute to the likelihood of consulting for physical symptoms $(1.44,1.09$, 1.91 ; other predictors were: physical symptoms that cut down activity $(2 \cdot 28,1 \cdot 8,2 \cdot 88)$; physical symptoms reported $(1.81,1.59$, $2 \cdot 07)$; long standing illnesses $(1 \cdot 34,1 \cdot 17$,
$1.53)$; social class $(1.12,1.02,1.25)$; age cohort $(1 \cdot 43,1 \cdot 06,1 \cdot 92))$.

Conclusions - The initially strong associations between gender and consulting for at least one of total number of symptoms and for at least one of the symptoms of malaise was explained quite simply by women reporting more of these symptoms. However, women were more likely to consult for at least one of the reported physical symptoms, even when other symptoms and illnesses and socioeconomic factors had been taken into account. These findings will be further explored.

Variations in general practitioner utilisation with occupational class and employment status

A T O FANI KAYODE, ${ }^{1}$ P C CRYER, ${ }^{1}$ Y DOYLE,${ }^{1}$ A MARYON-DAvIs ${ }^{2}\left({ }^{1}\right.$ South East Institute of Public Health; ${ }^{2}$ Lambeth, Southwark and Lewisham Health Commission, London)

Study objectives - The work aimed to estimate the occupational class variations in general practitioner utilisation rates.

Design - This was a population based, cross sectional postal survey using a self completion questionnaire.

Setting - The South East Thames Regional Health Authority region, which included Kent, East Sussex, and south east London (both inner and outer London).

Participants - Subjects included 24562 residents of south east Thames aged 16 and over in 1992 .

Main results - An average of $66 \%$ of those who reported impairments had visited their GP within the last three months; this compares with $36 \%$ for the unimpaired ( $p$ value $<0.0001)$. The GP utilisation rate varied mostly with the level of disability, age, and gender. Variation was also apparent with socioeconomic position, demography, lifestyle, and geographical location. After adjusting for other factors, no occupational class variation was found. A variation in GP utilisation rates, after adjusting for other variables, was found for employment status for both the impaired and the unimpaired groups, which was modified by sex $(p<0.0001)$ and by the type of impairment $(p<0.02)$ for the impaired, and solely by sex $(p<0.002)$ for the unimpaired.

Conclusions - This comprehensive and unique study found that for the impaired group, despite clear social inequalities in distribution of impairments and disability (that is lower occupational classes having a higher incidence of more severe disability), occupational class does not seem to create a "double disability" in utilisation of GP services. With respect to employment status the clear message that can be abstracted from this is that male utilisation is more for those outside the labour market.

Socioeconomic variations in GP consultations among older people

ELIZABETH BREEZE, ASTRID FLETCHER ( $D e$ partment of Epidemiology, London School of Hygiene and Tropical Medicine)

Objectives - To explore the socioeconomic/ demographic variations in GP consultation patterns among people aged 65 and over.

Design - The 1981 survey of morbidity in general practice in which records of all consultations were kept for a year in 48 selected general practices.

Setting - Twenty five general practices in England and Wales for which the data were linked to the 1981 census.

Subjects - A total of 21387 people aged 65 and over on the GPs' lists and living in the community.

Methods - Logistic regression was used to examine the influence of socioeconomic and demographic variables on rates for all consultations, those in specified locations, and those for specified groups of illnesses. Analyses were done separately for men and women and adjustments made for age and other confounding variables.

Results - About a quarter of older people did not consult during the year. A substantially higher percentage of those who consulted did so in the surgery $(88 \%$ of men and $82 \%$ of women) than at home ( $38 \%$ of men and $44 \%$ of women). About one third of older people consulted for a serious illness. Patients aged 75 years and over were more likely to have several consultations for serious illnesses than those aged 65-74 years; they were about three times as likely to have a rate of five or more consultations per year at home. Single people were less likely to consult at all than ever married people (OR for men $0.57,95 \%$ CI $0.48,0.68$; OR for women $0.7495 \% \mathrm{CI}$ $0.65,0.83$ ). The main risk factors for frequent consultations ( $\geq 10$ per year) were age, council tenure, and living in the north. Household car availability, living alone, lacking an inside toilet, and being in an urban area were relatively unimportant predictors of consultation rates. People in council tenure were more likely to have at least five home visits (OR for men 1.23, 95\% CI 1.04, 1.45; OR for women $1 \cdot 30,95 \% \mathrm{CI} 1 \cdot 15,1 \cdot 47)$ and to consult at least five times for "trivial" illness (OR for men $1.40,95 \%$ CI 1.19, 1.64; OR for women $1.48,95 \%$ CI $1.32,1.66)$. For women, council tenure was also a predictor for frequent consultations for serious illness and in the surgery.

Conclusions - Council tenure was a consistent predictor of the frequency, location, and nature of GP consultations by community dwelling people aged 65 years and over. These results provide evidence for the continuing influence of socioeconomic inequalities on health and service utilisation in old age.

Variations in general practice prescribing costs

D G COOK, ${ }^{1}$ F A MAJEED, ${ }^{1}$ N EVANS ${ }^{2}\left({ }^{1}\right.$ Department of Public Health Sciences, St George's Hospital Medical School, London; ${ }^{2}$ Merton, Sutton and Wandsworth FHSA, London)

Objectives - To investigate the relative importance of patient and general practice characteristics in explaining variations between practices in the mean net ingredient cost per patient and the average number of items dispensed per patient.

Design - This was an ecological study examining variations in prescribing among 131 general practices using prescribing analyses and cost (PACT) data and demographic and census derived variables for the practices.

Setting - Merton, Sutton and Wandsworth Family Health Services Authority, which covers parts of inner and outer London.

Main outcome measures - Mean net ingredient cost per patient in 1993-94 and the average 
number of items dispensed per patient in 1993-94.

Results - The mean net ingredient cost per patient varied from $£ 18.60$ to $£ 105.13$, and the average number of items per patient varied from 3.6 to 14.9 . Differences in prescribing had a large impact on prescribing budgets. For example, two practices, each with list sizes of 10000 patients, lying on the 25th and 75 th centiles respectively for net ingredient cost per patient would have total net ingredient costs that differed by $£ 157000$. In a forwards stepwise regression model, three variables explained $33 \%$ of the variation in net ingredient cost per patient and two variables explained $30 \%$ of the variation in the number of items per patient. The percentages of the practice population aged 65 to 74 years and aged 0 to 4 years were positive predictors of net ingredient cost per patient, and the percentage of items prescribed generically was a negative predictor of net ingredient cost per patient. The estimated percentage of the practice population with chronic illness was a positive predictor of the average number of items per patient, and the percentage of the practice population aged 25 to 34 years a negative predictor of the average number of items per patient.

Conclusions - Only about one third of the variation in prescribing costs between practices could be explained using routinely available data. A capitation based formula to allocate prescribing budgets would be difficult to implement because of the large changes that would result in general practice prescribing budgets. Population based research using data collected on individual patients and practices is required to: (1) improve our understanding of the patient and practice factors that influence prescribing; (2) help explain why prescribing costs and prescribing rates vary so widely between practices; (3) improve the current method of allocating prescribing budgets to general practices.

OTHER HEALTH SERVICES RESEARCH

\section{Modelling the effects of physical activity interventions}

B NAIDOO,${ }^{1} \mathrm{M}$ THOROGOOD, ${ }^{1} \mathrm{~K}$ MCPHERSON, ${ }^{1} \mathrm{~L}$ GUNNING-SCHEPERS ${ }^{2}$ ( ${ }^{1}$ Health Promotion Sciences Unit, London School of Hygiene and Tropical Medicine; ${ }^{2}$ Institute of Social Medicine, University of Amsterdam)

In the realm of health promotion it is not possible to evaluate the effectiveness of intervention strategies by experimental or observational methods since the time scales involved with longitudinal studies can be prohibitive. A partial solution to this problem is the use of computer models which allow policy makers to simulate the effects of intervention scenarios within a population, using available data on risk factor prevalence and the related relative risks of disease mortality, and so projecting the results over several generations.

One such computer model is Prevent. Developed by one of the authors (LG-S) and adapted by the health gain project to model an English and Welsh population, this simulates risk factor interventions for a population and produces results in terms of the reduction in mortality.

Lack of physical activity has been shown to be a strong independent risk factor for
CHD mortality. In addition, population surveys have shown that only a small percentage of the British population takes enough exercise to protect against CHD. Consequently the promotion of physical activity is currently the focus of much discussion.

Prevent has been used to provide information on the likely effects of possible targets for physical activity promotion. The three targets simulated were:

- To reduce the proportion of the population, aged 15 to 64 , who were sedentary by $25 \%$,

- To increase the proportion of the population, aged 15 to 64 , who were moderately active by $25 \%$,

- To increase the proportion of the population, aged 15 to 64 , who were vigorously active by $25 \%$.

We have looked separately at the effects of achieving these targets in men and in women, and in different age groups.

The simulations showed only a small change in the CHD death rate, with women achieving a much smaller reduction than men. Although in terms of actual years of life gained the targets achieved more significant results, with the second target postponing just over 12000 deaths after 25 years, this result would only be equivalent to a simulated $2 \%$ reduction in the smoking prevalence. The modelling also showed that the targets were more effective if they were concentrated on those people over 45 years of age as opposed to all, or younger age groups.

Overall the model provided useful information on the effectiveness of possible physical activity targets, helping to compare strategies to identify those which generate the most health gain.

UK study of urgent admission to hospital: basis for a charter standard?

R R WEST, M ROSEN (University of Wales College of Medicine, Cardiff)

Rather more than half of all hospital admissions are urgent or emergency ones. Contracting arrangements following the purchaser-provider separation emphasise the elective services and there are few guidelines or standards for the emergency services. The UK study of urgent or emergency admission to hospital, under the auspices of the Clinical Standards Advisory Group which advises UK health ministers, was designed to describe current practice and policy and their variations across the country, to identify best practice and policy and potential for the development of guidelines and standards, and to inform policy makers.

Thirty representative acute hospitals across the country were included in the study. All patients admitted urgently or as emergencies during one week were monitored by teams of doctors and nurses and all steps of the admission process were observed and timed and any delays or non-optimal management and their reasons were noted. Outcome at 28 days was assessed by clinical record review.

Wide variations in all "key" admission timings were observed: time from arrival in hospital to doctor attends $8 \%$ to $72 \%$ seen within 30 minutes, time to initial clinical action $43 \%$ to $91 \%$ within 1 hour, time to admission to ward $14 \%$ to $96 \%$ within 2 hours, time to definitive clinical management $32 \%$ to $91 \%$ within 3 hours. Wide variations were observed also in 28 day inhospital mortality $(2 \%$ to $10 \%)$ and timeliness and mortality were as- sociated, after adjustment for age, sex, speciality mix, and casemix.

These timings of the key stages of urgent admission, congruent with the qualitative assessment of expert hospital visitations, serve as useful proxies for quality of care for urgent admissions and provide a baseline on which to develop guidelines and standards.

Fastrak - the evaluation of an innovative approach to day surgery

ELAINE MCCOLL, MARK DEVERILL, MARTIN ECCLES, COLIN BRADSHAW, CHRIS PRITCHETT, TREVOR ARMITAGE (Centre for Health Services Research, University of Newcastle upon Tyne) Aims - Day surgery is increasingly being seen as a cost effective method of delivery for certain specified procedures. Traditionally, patients are referred to a hospital consultant for preoperative assessments in outpatient clinics. But for fit patients, there are potential savings to be made by having the investigations carried out by a general practitioner. The south Tyneside FASTRAK initiative was a pilot study for this method of referral. The impact of the initiative was evaluated in terms of waiting times for surgery and patient and professional satisfaction.

Methods - Information on patient characteristics, presenting diagnosis, surgical procedure, and referral and operation dates were abstracted from patients' hospital records. Data on patient satisfaction were collected using the Audit Commission's previously validated questionnaire. General practitioners' experiences and views of the service were sought using a structured self completion questionnaire sent to all general practitioners practising in the district six months after the introduction of the service.

Results - The main time saving for FASTRAK patients is in the time from referral to being placed on a waiting list. For patients undergoing inguinal hernia repair under conventional day case surgery, the mean time from referral to waiting list was 124 days, compared with 12 days for FASTRAK patients. Overall levels of patient satisfaction were high. However, FASTRAK patients were more likely to have received printed information about their treatment before going into hospital. Controlling for casemix, there was no significańt difference in reported complication rates between the two groups. General practitioners were also satisfied; $59.6 \%$ felt FASTRAK should be extended to other specialities. The service was seen to offer benefits to patients, most notably in terms of shorter waiting times. However, some barriers to using the service were also identified; chief amongst these was a lack of suitable patients.

Discussion - The FASTRAK service offers patients a much shorter waiting time on average. This is likely to provide benefit by minimising the period in which they suffer the impact of their condition. Nor do FASTRAK patients seem to be any less satisfied with the service they receive or experience any more complications. Finally, the service seems to be generally popular with general practitioners despite the existence of some barriers to referring patients in this way. These results suggest that FASTRAK offers an acceptable alternative to conventional day case surgery. Further information on the costs to patients of the two modes of service delivery should be collected in any further evaluations. Any 
extension of the FASTRAK service should also consider some modification of guidelines to widen its scope in terms of patient eligibility.

International variation in intervention rates: what are the implications for patient selection?

NICK BLACK, ${ }^{1}$ MARK E GLICKMAN, ${ }^{2}$ JIAO DING, ANN FLOOD ${ }^{3}$ ( ${ }^{1}$ Health Services Research Unit, London School of Hygiene and Tropical Medicine; ${ }^{2}$ Department of Health Care Policy, Harvard Medical School; ${ }^{3}$ Center for Evaluative Clinical Sciences, Dartmouth Medical School) Objectives - Are populations of countries with higher rates of use better off as a result of treating more people? Do low rate countries utilise a more rational selection process so that those people most likely to benefit receive treatment while those with little to gain do not?

Design - Modelling of retrospective data from population surveys, patient cohort studies, and population intervention rates. Comparison of Maine (USA) and Oxford/North West Thames health regions (OXNWT) in the UK

Subjects - Men aged 55 years and above in the population with urinary symptoms suggestive of benign prostatic hyperplasia and men undergoing surgical treatment.

Main measures - Ratio of probability of surgery in Maine to that in OXNWT by level of symptom severity.

Results - The rate of surgery in Maine was $54 \%$ higher than in OXNWT. A higher proportion of patients in Maine had no or only mild symptoms before surgery $(22 \% v 11 \%$; $\mathrm{p}<0.0001)$. The probabilities of being operated on in a given year varied by symptom severity in both areas. The probability was significantly higher in Maine for men with no or mild (ratio $=3.05 ; p<0.0001$ ) and lowmoderate symptoms (ratio $=1.64 ; \mathrm{p}<0.0001$ ) but not for those with high-moderate (ratio $=$ $1 \cdot 27 ; \mathrm{p}=0.03$ ) or severe symptoms (ratio $=$ $1.10 ; \mathrm{p}=0 \cdot 28)$. These persisted on testing the sensitivity of the key assumptions.

Conclusion - Some of the international variation in intervention rates arises because of differences in the level of inappropriate care: men in Maine were no better off, because a more rational selection process tended to be used in the UK.

CANCER

Childhood cancer in Muslim and nonMuslim Asians: is parental consanguinity responsible?

J E POWELL, ${ }^{1}$ A $M$ KELLY, ${ }^{1}$ S E PARKES, ${ }^{1}$ T R P COLE, ${ }^{2}$ J R MANN ${ }^{1}$ ('West Midlands Regional Children's Tumour Research Group, The Children's Hospital, Birmingham; ${ }^{2}$ Clinical Genetics Unit, Birmingham Maternity Hospital)

Objective - An investigation to determine whether parental consanguinity increases the risk of childhood cancer in West Midlands Asians.

Design - Cancer incidence rates were derived for Asian Muslim (high prevalence of consanguinity) and non-Muslim (low prevalence of consanguinity) children resident in the West Midlands Regional Health Authority area. Congenital abnormalities in these cancer patients were also investigated.

Subjects - All Asian children 15 years of age, with a diagnosis of cancer made between 1978 and 1992 , who were resident within the study area.

Results - Between 1978 and 1992, 187 Asian children with cancer were registered. Of these, 54\% were Muslims, mainly from Pakistan and Bangladesh. Cancer incidence was significantly higher in Pakistani/Bangladeshi Asians, with an age standardised incidence of 163 cases per million per year, compared with 115 for Indians and 125 for Whites. An excess of solid tumours was found in the Pakistanis, while both Asian groups had a high incidence of lymphoma. Among Asian cancer patients, congenital malformations were significantly more common in the Muslims $(21 \%)$ than in the non-Muslims (7\%). Eight per cent of the Muslims suffered from autosomal recessive conditions and $5 \%$ had autosomal dominant disorders. The proportion with sporadic genetic conditions was similar in both groups. Of the Muslims with malformations, $48 \%$ had cancer malformation/ predisposition syndromes, and in 33\% of cases there was a relative with both a cancer and a malformation.

Conclusions - Genetic factors contribute significantly to the cancer excess in Muslims, inherited genes causing both malformations and cancer. Consanguinity contributes to the prevalence of cancers related to autosomal recessive disorders. However, many cancers are linked to autosomal dominant disorders, which may be a consequence of older paternal age at conception, in this or previous generations.

Radiotherapy, alkylating agents and the risk of bone cancer after childhood cancer

M M HAWKINS, ${ }^{1}$ L M KINNIER WILSON, ${ }^{1} \mathrm{H}$ S BURTON ${ }^{1} \mathrm{M}$ H N POTOK, ${ }^{1} \mathrm{D}$ L WINTER, ${ }^{1} \mathrm{H} \mathrm{B}$ MARSDEN, ${ }^{2}$ M A STOVALL ${ }^{3}$ ( ${ }^{1}$ Childhood Cancer Research Group, University of Oxford; ${ }^{2}$ Royal Manchester Children's Hospital; ${ }^{3}$ M D Anderson Cancer Center, University of Texas, USA)

Objective - To investigate the incidence and aetiology of second primary bone cancer after childhood cancer in Britain.

Design - Cohort study and case-control study.

Setting - Britain and the population based national registry of childhood tumours.

Subjects - Cohort of 13175 three year survivors of childhood cancer diagnosed in Britain between 1940 and 1983, among whom 55 subsequent bone cancers were diagnosed. A case-control study of 59 cases developing second primary bone cancer; 220 controls were selected matched for sex, type of first cancer, age at first cancer, and interval to diagnosis of subsequent bone cancer.

Main outcome measures - Cumulative risk of bone cancer subsequent to childhood cancer Cumulative dose of radiation received at the site of the second bone cancer in the case and at the corresponding anatomical site in the matched controls as a result of radiotherapy. Cumulative dose $\left(\mathrm{mg} / \mathrm{m}^{2}\right)$ of alkylating agents and vinca alkaloids received as part of anticancer chemotherapy.

Results - The percentage of three year survivors developing bone cancer within 20 years did not exceed $0.9 \%$, except for those originally diagnosed with heritable retinoblastoma, Ewing's sarcoma, and other malignant bone tumours for whom the cor- responding risks were $7 \cdot 2 \%, 5 \cdot 4 \%$, and $2 \cdot 4 \%$ respectively. The risk of bone cancer increased substantially with increased cumul ative dose of radiation which bone had received during radiotherapy for the original childhood cancer. However, at the highest levels of exposure the risk appeared to decline somewhat. Exposure to less than $10 \mathrm{~Gy}$ was, at worst, associated with only a small increased risk of bone cancer. The risk of bone cancer increased with increased cumulative dose of alkylating agents, but this association was considerably weaker than that with radiation exposure.

Conclusions - This population based study provides grounds for reassuring the majority of survivors $(89 \%)$ that their risk of developing bone cancer within 20 years of three year survival is less than $1 \%$. The higher risks of bone cancer after specific rare types of childhood cancer provide a rational basis for surveillance. The relative risks of bone cancer after specified levels of exposure to radiation should help in decisions of whether to include radiotherapy in future treatment protocols for childhood cancers.

Cancer in the offspring of survivors of childhood leukaemia and non-Hodgkin lymphomas

D L WINTER, M M HAWKINS, G J DRAPER (Childhood Cancer Research Group, University of $O x$ ford)

Understanding the extent to which childhood leukaemia and non-Hodgkin lymphomas are heritable is important to the survivors of these diseases, their families, and the clinicians who provide genetic counselling. Such understanding is also relevant to the possibility raised by Gardner et al (BMF 1990; 300 423-9) that paternal preconception irradiation may be an aetiological factor in these diseases. No malignant neoplasm was diagnosed among 382 offspring of survivors of childhood leukaemia and non-Hodgkin lymphoma followed up for a median period of 5.8 years; the largest available cohort of such offspring. These data indicate that it is unlikely that the risk of a malignant neoplasm occurring in the offspring exceeds eight times that expected in the general population. Similarly, the risk of leukaemia and non-Hodgkin lymphoma among offspring is unlikely to exceed 21 times that expected. The proportion of survivors of childhood leukaemia and nonHodgkin lymphoma with the heritable form of these diseases is unlikely to exceed $5 \%$, assuming an autosomal dominant pattern of ransmission, with penetrance of at least $70 \%$ and that all heritable cases develop by age 15 years. The best (that is, at present most likely) estimates of these risks are of course much lower. There was no evidence of an excess of congenital abnormalities among the offspring and the sex ratio was similar to that expected from the general population.

Does early respiratory environment influence lung cancer risk? Evidence from studies in the west of Scotland

D J HOLE, C R GILLIS, G $M$ WATT (West of Scotland Cancer Surveillance Unit, Glasgow; Department of General Practice, University of Glasgow)

Glasgow has one of the highest rates of lung cancer in the world. We have previously 
shown that for equal numbers of cigarettes smoked the risk of lung cancer is twice that found in other major cohort studies. This presentation draws together evidence from three local studies to try to explain this difference.

The first two studies consider the role of air pollution. One is based on modelling the rates of lung cancer on a small area basis as a function of cigarette smoking, air pollution, asbestos exposure, and socioeconomic status. Direct measures of air pollution and socioeconomic status are available and proxies for cigarette smoking (chronic bronchitis mortality) and asbestos exposure (mesothelioma incidence) are used. A significant relation between air pollution and lung cancer incidence exists for both males and females after adjustment for the other three factors listed. One of the more stringent criteria for accepting causalty is whether risk declines when the risk factor is reduced. The second study examines the trend in lung cancer incidence in Glasgow following the implementation of the Clean Air Act in 1959 and compares it with the same data for rural areas (control). No convergence of the two trends is apparent in the 33 years up to 1992 . This suggests an association which is not directly causal.

A third study, the Renfrew/Paisley cohort where the general population live close to each other (only small variation in air pollution) also identifies factors in addition to cigarette smoking which are related to increases in lung cancer incidence. These include a low $\mathrm{FEV}_{1}$, presence of phlegm, wheezing, a positive response to tuberculin testing, and social class.

Why should all these factors together with air pollution show an association with increased lung cancer risk which is additional to the effect of cigarette smoking? The theory proposed is that these factors are, or represent, a response to a poor respiratory environment which is present both inside (for example, damp housing, overcrowding, passive smoking) and outside (air pollution) the home. Lung development is most sensitive to external factors during young ages. Today's lung cancer patients were born in the 1920 s and 1930 s, precisely when housing and pollution conditions were very poor in urban west of Scotland. It is suggested that the damage done to the lung at this time is such that subsequent exposure to a carcinogen (tobacco, asbestos) makes the occurrence of lung cancer more likely. This explanation implies that the high rates observed locally are not a "west of Scotland" effect but a deprivation effect. Support for this has come from a comparison with manual Whitehall civil servants who exhibit similar levels of lung cancer to manual Renfrew/Paisley subjects.

PREGNANCY AND CHILDHOOD (II)

\section{Is there too much intervention during labour?}

F L R WILLIAMS, ${ }^{1}$ C DU V FLOREY, ${ }^{1}$ V R TINDALL, ${ }^{2}$ N B PATEL, ${ }^{3}$ P W HOWIE ${ }^{3}$ ( ${ }^{1}$ Department of Epidemiology and Public Health, and ${ }^{3}$ Department of Obstetrics and Gynaecology, University of Dundee; ${ }^{2}$ Gainsborough House, Manchester) Introduction - Interventions during labour should be avoided unless there are clear indications to the contrary, yet there is a general view among women, midwives, and obstetricians that there are too many interventions during this time. There are few systematic data on this subject.

Objective-To determine whether intervention during labour in primigravidae exceeds or is less than that recommended in hospital guidelines.

Design - Cross sectional survey of women delivering in NHS hospitals in the UK.

Subjects and methods - One hundred and one hospital maternity units were randomly selected from a total of 226 hospitals. Forty consecutive low risk primigravid women were recruited in each hospital. Detailed information was collected about the number and reasons for seven broad categories of intervention: caesarean section, type of delivery, vaginal examination, episiotomy, augmentation of labour, fetal monitoring, and pain relief. Information from guidelines for the management of normal labour was obtained from each collaborating hospital.

Results - Ninety eight hospitals participated in the study and information was collected on 3160 women (response rate of $81 \%$ ). After one letter of reminder 95 hospitals $(97 \%)$ sent in details and/or written guidelines about their management of normal labour. Sixteen specific recommendations were identified that were common to all guidelines. This enabled a comparison between hospital practice and guideline recommendations within the categories: type of delivery, vaginal examination, augmentation of labour, and fetal monitoring. There was marked discrepancy between hospital guidelines and practice in four areas. The guidelines recommended that vaginal examinations should be four hourly; after adjustment for duration of labour almost $75 \%$ of the women surveyed had more vaginal examinations than expected. A little over one quarter $(27 \%)$ had between three and eight more examinations than expected. Two procedures were used much less frequently than recommended - baseline fetal cardiotocographic monitoring and restriction of the second stage of labour in the absence of epidural analgesia. Only $21 \%$ of women surveyed had a baseline reading recorded. Most women in the absence of epidural analgesia had a restricted second stage of labour but in $10 \%$ it lasted over two hours. Finally, although there was little consensus on when artificial rupture of membranes should be performed, it was used on $53 \%$ of the women. In the remaining 12 recommendations the survey found slightly less intervention than recommended in the guidelines.

Conclusions - Apart from vaginal examinations, this study provides little support for the belief that there is too much intervention during labour. There was substantially less intervention than recommended for two procedures which raises the possibility of under intervention. Implications for the service will be discussed.

Effects of socioeconomic and psychological factors, smoking, alcohol, and caffeine on preterm birth

J L PEACOCK, J M BLAND, H R ANDERSON ( $D e-$ partment of Public Health Sciences, St George's Hospital Medical School, London)

Objective - To investigate the relationships between preterm birth and socioeconomic and psychological factors, smoking, alcohol, and caffeine consumption.

Design - Prospective study.
Setting - District general hospital in inner London.

Participants - A series of 1860 white women booking at St George's. Exclusions were due to multiple pregnancy, diabetes, refusals, and loss to follow up, leaving a sample of 1513 women.

Measurements - Gestational age was calculated from the last menstrual period and ultrasound data. Preterm birth was defined as less than 37 completed weeks. Explanatory variables included smoking, alcohol, and caffeine consumption, and a range of indicators of socioeconomic status and psychological stress.

Main results - Unifactorial analyses showed that lower social class, less education, single marital status, low income, trouble with "nerves" and depression, help from professional agencies, and little contact with neighbours were all significantly associated with an increased risk of preterm birth. There were no effects of smoking, alcohol, or caffeine on the length of gestation overall but an association was observed between smoking and delivery before 32 weeks. Cluster analysis highlighted three subgroups of women delivering preterm. Clusters 1 and 2 were mainly women of low social status. The third cluster comprised older, non-smokers with higher social status. Mean gestational age was highest in the third group.

Conclusions - Adverse social circumstances are associated with preterm birth but smoking is not, apart from an association with very early births. This is converse to the findings for fetal growth (birthweight for gestational age) obtained in the same study where a strong effect of smoking was observed but there was no evidence for any association with psychosocial factors. The risk factors for the two components of birth weight (fetal growth and gestational age) are therefore different. This has implications for the interpretation of variations in birth weight between populations and for public health policy. In addition an understanding of factors affecting these two components may provide clues about possible mechanisms for observed associations between birth weight and disease in later life.

The social origins of infantile colic: risk factors identified in the Sheffield child development study

N S CROWCROFT, D P STRACHAN (Department of Public Health Sciences, St. George's Hospital Medical School, London)

Background - Infantile colic is commonly reported by parents but is poorly understood. It may contribute to postnatal depression, non-accidental injury, and delayed development of the child. Despite conflicting results from intervention studies, the advice given to parents by health care professionals is often to change the feeding practice in some way.

Objective - To explore risk factors for parental reporting of infantile colic during the first month of life.

Methods - All 76508 infants born in Sheffield between August 1975 and May 1988 were followed up at 1 month of age by health visitors. Information was collected on infantile colic, socioeconomic characteristics of the family, mode of feeding, and behaviour of the infant. Hypotheses relating infant colic with the method of feeding, parental characteristics, and material circumstances of the 
family were tested in a logistic regression model using SAS and GLIM4 statistical software.

Results - Information on infantile colic was available on 72995 (95\%) infants. The period prevalence of colic in the first month of life was $18 \%$. In univariate analyses, associations were found with increased reporting of colic and: higher parental age; lower maternal parity; non-manual occupation; breast feeding; higher parental age at completing full time education; more affluent district of residence; and better state of repair of the home. Babies who were reported to be colicky were no different from non-colicky babies in sex or gestational age at birth. In a logistic regression analysis, the association with socioeconomic factors remained significant, with a higher prevalence of infantile colic in more affluent areas and homes. Method of feeding was not an independent risk factor, although parents of colicky babies changed the mode of feeding more often during the first month than parents who did not report any colic.

Conclusions - In this statistically powerful study, the method of infant feeding was not a significant risk factor after controlling for confounding variables, supporting the findings of those intervention studies which have found that dietary manipulation helps in very few cases. The most effective form of management of infantile colic by primary health care teams may be parental education about normal infant behaviour.

The relationship between the treatment of cough during early infancy and the mothers' educational qualifications

N S HAWKINS, C R DEWEY (Institute of Child Health, Royal Hospital for Sick Children, Bristol)

Method - The Avon longitudinal study of pregnancy and childhood (ALSPAC) is a longitudinal cohort study designed to investigate environmental factors associated with the health of children. The study is based in the three health districts of Avon in the United Kingdom. The study population comprises pregnant women whose expected dates of delivery were between 1 April 1991 and 31 December 1992 and their children. Self completion questionnaires are posted at regular intervals to women participating in the study. The "six month" postnatal questionnaire included enquiries about the infants' symptoms and administration of drugs during the first six months of postnatal life. The "32 week" antenatal questionaire, administered during pregnancy, included enquiries about the educational qualifications of the mother.

Results - This analysis concerns 10867 cases where both questionnaires had been completed. The proportion of infants with cough symptoms was not associated with maternal educational level (Pearson $\chi^{2}=0.5, p=0.97$ ), ranging from $64 \%$ to $65 \%$. However, the proportion of infants visiting a doctor because of cough symptoms was highly and progressively associated (Pearson $\chi^{2}=65$, $\mathrm{p}<0.00001)$ with maternal educational level, ranging from $44 \%$ of infants with mothers in the lowest educational level group to $33 \%$ of infants with mothers in the highest group. Similarly, the proportion of infants receiving cough medicine progressively varies from $20 \%$ of infants whose mothers were in the lowest educational group to $10 \%$ of children with mothers in the highest group (Pearson $\left.\chi^{2}=201, p<0.00001\right)$. The proportion of infants with cough symptoms lasting for more than two days was also associated with educational level (Pearson $\chi^{2}=11, p=0.02$ ). However, the proportion with such symptoms, which ranged from $42 \%$ to $47 \%$, was highest in children whose mothers were in the highest educational group. This, in addition to the consistency of the proportion of children $(64 \%$ to $65 \%)$ with any cough symptoms, suggests that the variation in treatment was not due to variation in severity of symptoms.

Summary - It seems that mothers with lower educational qualifications are significantly more likely to seek medical advice if their children have cough symptoms and are more likely to treat their children with cough medicine. The magnitude of the difference between the different groups of mothers is striking: half as many children receiving cough medicine and two thirds as many visiting the doctor for cough symptoms in the highest maternal educational group compared with the lowest. These findings have significance for the epidemiological interpretation of general practice based records.

ENVIRONMENT AND BEHAVIOUR

Ill health and housing costs: analysis of the British household panel survey

JIM CONNELLY, DAVID BRAUNHOLTZ, JENNY BARRETT, ALAN S RIGBY, MIKE ROBINSON (Division of Public Health, Nuffield Institute for Health, University of Leeds)

There is increasing recognition that ill health is not only caused by poor housing conditions but that it may also restrict housing opportunities.

Though, in theory, ill health should not determine housing quality - because of the health role of UK housing policy - in practice it is often found to do so.

The crucial link in this health-housing model is the impact of ill health on the ability to meet housing costs. We present evidence from an analysis of the first wave of the British household panel survey (BHPS) which has examined the relationship between ill health and problems with meeting housing costs The results confirm that local authority and housing association tenants are much more likely than other groups to have experienced a health problem which has influenced the work they can do ( $23 \% v 9 \%$; $<0.01)$. Moreover, those with health problems have experienced significantly more problems with housing costs during the previous year $(\mathrm{p}<0.01)$. When a logistic regression model was used to adjust for possible confounding by age, adjusted income, household type, registered disability, and gender there was still a significant influence of health problems on ability to pay. The patterning of such problems suggests that current and announced changes in housing policy will only exacerbate an already unsatisfactory situation.

\section{Workplace health or worker health - health effects of the physical en-} vironment in offices

A F MARMOT, J ELEY, M NGUYEN, E WARWICK, M G MARMOT (Department of Epidemiology and Public Health, University College London Medical School)
Aim - To establish the relative importance of the physical and psychosocial work environment in the occurrence of symptoms associated with the "sick building syndrome" (SBS).

Background - SBS is described as a group of symptoms which people experience while they are in specific buildings. 'Investigations have mostly taken place in office buildings. It is claimed that aspects of the physical environment cause the symptoms and that the symptoms cause sickness absence. Isolating particular features responsible for the symptoms has proved elusive - SBS is thought to have multiple causes acting together in different combinations. The causes identified vary between studies, but usually include the ventilation system, the thermal environment, indoor air quality, cleanliness, work characteristics and psychosocial factors.

Design - Cross sectional study of social, psychological, and lifestyle influences on health of office workers in the Whitehall II study. During 1991 to 1993 , questions on the office environment were added to the main questionnaire completed by 6831 people still in employment. Fieldwork was conducted in 289 workstations drawn as a stratified random sample within 29 buildings in which large samples of the study population worked. Less detailed data were gleaned on a further 15 buildings.

Subjects - Male and female civil servants aged 38 to 62 years.

Results - No significant relationship was found between any aspect of the physical work environment and symptom prevalence, with the exceptions of relative humidity, inhalable dust, being able to exercise some control over light, heat, and ventilation in the workplace, being close to a window, and possibly the amount of total volatile organic compounds in the air. Stronger relationships were found with features of the psychosocial work environment including low variety and skill use, high work pace, low support, and low control, and with scores on the general health questionnaire. Exposure to passive smoking at home or work and a long journey to work were also significantly associated with symptom prevalence.

Conclusion - The physical environment of workstations and office buildings seems to have a less significant effect on symptom prevalence than features of the psychosocial work environment, passive smoking, and the length of the journey to work.

1 Raw G. Sick building syndrome: a review of the evidence on causes and solutions Health and Safety Executive Research Report No. 42/1992. London: HMSO, 1992.

What is the impact of taking account of occupational grade on gender differences in health?

C EMSLIE, K HUNT, S MACINTYRE (MRC Medical Sociology Unit, Glasgow)

Objective - Relationships between gender, work, and health have often been explored using male and female employees working in very different jobs. We examine whether gender differences in health remain when men and women working full time in the same industry and within similar grades are compared.

Design - A questionnaire was sent to employees of a large Scottish bank. Where pos- 
sible, questionnaires were sent to equal numbers of men and women at each grade. Subjects - Altogether 1119 male and 1081 female bank employees responded $(76 \%$ response rate), across clerical, supervisory, and management grades.

Main outcome measures - Respondents indicated which of a list of 20 symptoms (subdivided into physical and malaise symptoms) they had experienced in the last month. They were also classified in terms of their consumption of alcohol (none, moderate, and excessive by Royal College of Physicians definitions) and tobacco (none, light, and heavy smokers).

Results - A female excess was highly significant for mean scores of all symptoms, and for physical and malaise symptoms, and a male excess for drinking. However, when symptoms were considered individually, fewer than half showed significant gender differences. When the measures were considered for employees at the three different grades, clerical workers showed a highly significant female excess for mean scores of total symptoms, physical and malaise symptoms; supervisors had a significant female excess for total symptoms and physical symptoms, but not for malaise symptoms, while managers had no significant gender differences. Clerical and supervisory workers, but not managers showed a significant male excess for drinking. There were no significant gender differences for smoking, either overall for men and women or within grades.

Conclusion - Most studies cannot control adequately for paid work when examining gender differences in health. This study reveals that examining gender differences for employees working within similar grades in the same organisation shows a much more complex pattern of gender differences than might be expected.

\section{New methods for identifying oc- cupational groups at increased risk using routinely collected data}

L M CARPENTER, ${ }^{1}$ N MACONOCHIE, ${ }^{2}$ E ROMAN, ${ }^{2}$ D $\mathrm{R} \operatorname{cox}^{3}\left({ }^{1}\right.$ Department of Public Health and Primary Care, University of Oxford; ${ }^{2}$ Cancer Epidemiology Unit, ICRF, Oxford; ${ }^{3}$ Nuffield College, Oxford)

In the UK, routinely collected national data have long been used to identify occupational groups at increased risk of adverse health outcomes. The analysis and interpretation of such data is not straightforward, one problem being that when examining associations simultaneously for several specific health outcomes across many occupational groups it is not obvious how best to identify those requiring further investigation since some may be high, or low, by chance alone. We have therefore developed a new methodological approach that attempts to deal with this problem. The methodological approach involves using standard analysis of variance techniques followed by an empirical Bayes procedure. Anomalous cancer-occupation associations can be seen as outliers in a plot of the resultant relative risk estimates.

In order to demonstrate the ability of the new methods to identify occupational groups at increased risk, they were applied in the analysis of over 250000 cancers registered in men in England between 1981 and 1987. Specific attention was given to two cancers for which occupational associations are well established, namely cancers of the nose and nasal sinuses and bladder cancer. The analyses clearly identified three occupational groups at increased risk of nasal cancers"other woodworkers", "cabinet makers", and "woodworking machinists". For bladder cancer an excess was found in rubber manufacturers.

That each of the occupational associations identified by these analyses is already well established demonstrates that the new methods are capable of detecting occupational groups at increased risk. We are now proceeding to apply the methods to the analysis of all other specific cancers in men, and of cancers in women. Selected results of these analyses will be presented and some conclusions will be made.

Suicide - an indicator of mental health or a symptom of deprivation?

P MCLOone (Public Health Research Unit, University of Glasgow)

Objective - To describe variation in Scottish suicide rates in relation to age, sex, the main geographical regions of Scotland, and postcode sectors categorised by socioeconomic census variables.

Main outcome measures - Age standardised mortality rates for deaths attributed to suicide (ICD E950-959) and deaths resulting from injury undetermined whether accidentally or purposefully inflicted (ICD E980-989).

Results - Between 1981 and 1993 male suicide rates rose while female rates fell. When examined by age, the rate among men aged 30 and over remained relatively constant, and the overall rise in male suicides was due to a $80 \%$ increase in rates at ages $15-29$. The increase in young male suicides was mainly from deaths by self poisoning and hanging which contributed $79 \%$ of the rise. The overall fall in female suicides was due to a $27 \%$ decrease in the rate among those aged 30 and over. This fall was largely a result of reductions in deaths from self poisoning and submersion. Suicide rates for women aged 15-29 increased during the period and had a relative rise similar to that shown by young men (92\%); young adults of both sexes experienced an increase in deaths from hanging and suffocation, gassing by vehicle exhaust fumes, and self poisoning. Both sexes at all ages showed an increase in poisoning from motor vehicle exhaust fumes, and poisoning from analgesics, antipyretics, and antirheumatics. These changes contrast with reductions in deaths from tranquilliser and sedative poisoning. Higher suicide rates among men in the Highland region relative to Scotland were a result of excess deaths from motor vehicle exhausts, firearms, and drowning. These higher rates could reflect the availability of these methods in rural areas, and not necessarily indicate a higher prevalence of individuals with a predisposition to suicide. Comparison of suicide rates in postcode sectors classified as affluent or deprived in 198 and in 1991 showed higher rates in deprived areas for both males and females. However, higher rates of poisoning by car exhaust fume were observed in affluent areas. Compared with 1980-82, rates for young adults were higher in all deprivation categories by 1990-92 but the increase was greatest in more deprived areas, where rates were more than twice those experienced in affluent localities.
Conclusions - Suicide rates reveal complex patterns when individual methods are examined. Part of the difficulty in understanding these patterns is in untangling variations due to change in the availability and choice of method as opposed to more relevant changes in the prevalence of individuals with a predisposition to suicide. Change in suicide rates, therefore, cannot be interpreted in simple ways as an indicator of change in the mental health of populations. However the incidence and increase in suicide rates among young adults was more pronounced within deprived localities, and this pattern cannot easily be explained by issues relating to opportunity. This observation presents a worrying trend and prompts questions about the role of social policy in reducing ill health and death caused by the incidence of mental illness in these localities.

\section{Could GPs do more to prevent suicide?}

FRANCES HASTE, ${ }^{1}$ JOHN CHARLTON, ${ }^{1}$ RACHEL JENkINs, ${ }^{2}$ ('Office of Population Censuses and Surveys, London; ${ }^{2}$ Department of Health, London)

Objective - To identify in suicide cases and matched controls: (a) the patterns of consultation, the frequency of diagnosis and treatment of mental illness, and the frequency of recording of known risk factors for suicide; and (b) whether other risk factors for suicide could be identified from GP medical records. Rationale - Reduction of suicide was one of the targets of the government's Health of the Nation strategy (1992), with the suggestion that the role of the GP in prevention could be enhanced. The potential for prevention by GPs will depend on (a) whether or not the patient has seen the GP, (b) whether the GP detects the risk of suicide, and (c) whether possibly preventive treatment is subsequently provided.

Methods - A case-control study was conducted by examining the computerised medical records of suicide cases and matched controls held in the general practice research database (formerly the VAMP database, containing 3.4 million patient records). We examined the prevalence of major disease, diagnosis of and treatment or referral for mental illness, frequency of recording of recent life events, and consultations with the GP in the 12 months before death.

Results - Preliminary results of 254 cases showed that $80 \%$ of suicide cases were male and $50 \%$ of cases had a history of diagnosis and/or treatment for mental illness. Major disease other than mental illness was similar in cases and controls, as was recording of major life events. However, the recording of major life events was very low, particularly in men. Suicide cases were more likely than controls to have consulted their GP in the week, month, and 6 months before death but nearly a third had not consulted their GP in the 6 months before death. Women who had consulted were more likely than men to have received a diagnosis of, and medication for, mental illness. Most women who had consulted had received drug treatment for mental illness but half of the men who consulted were not receiving treatment. This difference suggests that GPs were less likely to identify and treat mental illness in male patients at risk of suicide and this may also partly explain the large sex difference in successful suicide. 
Are young unemployed men at greater risk of future illness, even before they experience any unemployment?

SCOTT M MONTGOMERY, ${ }^{1}$ MEL J BARTLEY, ${ }^{1}$ DEREK G COOKE, ${ }^{2}$ MICHAEL E J WADSWORTH ${ }^{3}$ ('SSRU, City University, London; ${ }^{2} S t$ George's Hospital Medical School, London; ${ }^{3} M R C$ National Survey of Health and Development, University College London)

Objective - To examine the hypothesis that young men who become unemployed are more vulnerable to future illness even before they experience that unemployment.

Design - Longitudinal birth cohort study. Setting - Great Britain.

Subjects - A total of 2600 men with complete data from the national child development study (NCDS). The NCDS has collected data on all men and women born in one week in 1958, who were followed up using interviews, self completion questionnaires, and medical examinations at birth and at ages $7,11,16,23$ and 33 years.

Main measures - Amount of unemployment experienced in early adult life up to age 32 years was the outcome measure used. Exposure measures to indicate vulnerability to future ill health were: height at age 7 years and the Bristol social adjustment guide (BSAG) at age 11 years. The BSAG is a measure of behavioural maladjustment.

Results - Short stature at age 7 years and poor social adjustment were related to poor material circumstances in childhood and lower educational attainment. Men with short stature and poor social adjustment in childhood were more likely to experience unemployment in adult life, even when controlling for parental height, socioeconomic background, and education. These differences remained when men with preexisting illness were excluded from the analysis. The adjusted relative odds for unemployment risk associated with childhood height are 0.95 (95\% CI $0.92,0.97)$. These odds represent the decrease in risk of unemployment associated with each additional $\mathrm{cm}$ of height. The adjusted odds representing the increase in risk associated with each additional BSAG symptom are 1.03 (95\% CI 1.01, 1.04). Adult height was not significantly associated with unemployment. Conclusions - The relationship between unemployment and poor health arises, in part, because men who become unemployed are more likely to have accumulated risks to health during childhood. For them, the experience of unemployment may represent an additional set of health risks.

\section{Which smokers are helped to give up} smoking using the nicotine patch?

P L YUDKIN, L JONES, T LANCASTER, G H FOWLER (ICRF General Practice Research Group, Department of Public Health and Primary Care, University of Oxford)

Background - Nicotine replacement therapy is effective in helping people to give up smoking. The three forms now available - the transdermal patch, nicotine gum, and nasal nicotine spray - deliver nicotine at different rates and to different levels. It might therefore be expected that smokers with different characteristics, and with different degrees of nicotine dependence, would be helped more by one or other method.
Objective - To examine whether the effectiveness of the nicotine transdermal patch is related to nicotine dependence or to other smoker characteristics. To investigate factors predicting smoking cessation using the patch Design - Retrospective analysis of data from a double blind placebo controlled randomised trial of the transdermal nicotine patch.

Subjects - A total of 1686 patients recruited from 19 practices in Oxfordshire.

Main outcome measures - Continuous smoking cessation from 8 weeks to 52 weeks after the start of patch use, biochemically validated at 12,24 , and 52 weeks. The effectiveness of the patch was measured by the relative odds of sustained cessation using the nicotine patch compared with the placebo patch

Results - The patch was more effective in smokers with moderate nicotine dependence (odds ratio (OR) $1.94 ; 95 \%$ CI $1.24,3.04$ ) than in mildly or highly dependent smoker (OR $0.98 ; 95 \%$ CI $0.58,1.65$ ) ( $p$ value for difference in odds ratios $<0.05$ ) and more effective in those aged less than 50 years (OR $1.89 ; 95 \%$ CI $1.24,2 \cdot 87$ ) than in older smokers (OR 0.88; 95\% CI 0.49, 1.59) (p value for difference in odds ratios $<0.05$ ) Abstinence from smoking in the first week of the trial was the strongest predictor of sustained cessation, and was more common among smokers using the nicotine than the placebo patch (32.9\% compared with $21.6 \%$ $\mathrm{p}<0.0001$ ). Of first week abstainers, $24.9 \%$ and $28.1 \%$ in the nicotine and placebo group respectively achieved sustained cessation compared with $3.9 \%$ and $2 \cdot 1 \%$ of first week smokers.

Conclusion - The nicotine transdermal patch is most effective for smokers with moderate nicotine dependence and for younger smokers. Early abstinence from smoking is the strongest predictor of sustained cessation. A week's trial of the patch proceeding to longer term use if abstinence is achieved may be a cost effective policy.

\section{CARDIOVASCULAR EPIDEMIOLOGY}

The risk of serious disease among oral contraceptive users

P HANNAFORD, C KAY (Royal College of General Practitioners' Manchester Research Unit)

Introduction - Even though long term all cause mortality among pill users seems to be similar to that among never users, oral contraceptive use might still be associated with an important increased risk of serious but non-fatal illness. Objectives - To estimate the long term balance of serious illness among pill users.

Methods - Since the late 1960s, the Royal College of General Practitioners' oral contraception study has observed an initial cohort of 23000 pill users and 23000 non-users in order to determine the effects of oral contraceptives. Illnesses included in this analysis were those that are potentially life threatening and/or associated with long term disability, and which have been found (or postulated) to be associated with pill use: heart disease; stroke; peripheral vascular disease; thromboembolic disorders; cancer of the large bowel, rectum, liver, gallbladder, skin, breast, cervix, endometrium, ovary, pituitary, and central nervous system, unknown origin; liver disease; diabetes mellitus; multiple sclerosis; inflammatory bowel disease; rheumatoid arthritis. By September 1993, ever users had experienced 2132 first events during 312967 women years of observation and never users 1360 events during 214631 women years. The event rates in each group were directly standardised for age, parity, social class, and smoking, after events and periods of observation relating to pregnancy were excluded. The effects of older and newer pill formulations were estimated by grouping the pills by their oestrogen content.

Results - The overall relative risk (RR) between ever and never users was $1 \cdot 14$ (95\% confidence interval (CI) $1.06,1 \cdot 21)$. The elevated risk was restricted mainly to women who smoked: compared with non-smoking never users, non-smoking ever users RR 1.09 (95\% CI $0.99,1.20)$, smoking never users $1 \cdot 14(95 \% \mathrm{CI} 1 \cdot 02,1 \cdot 77)$, smoking ever users RR 1.36 (95\% CI 1.24, 1.49). Parity, social class, and age did not seem to materially affect the risk estimates between ever and never users. An inverse trend with oestrogen content was apparent among current users (oestrogen content $>50 \mu \mathrm{g}$ : RR $1.69,50 \mu \mathrm{g}$ : RR 1.47, <50 $\mu \mathrm{g}$ : RR 0.89; trend test $\chi^{2} 12 \cdot 3$, $1 \mathrm{df}, \mathrm{p}<0.01$ ).

Conclusion - Pill users have a slightly increased long term risk of serious illness, an effect which is concentrated mainly in women who smoke. The more recently introduced ora contraceptives have a lower risk potential than older preparations.

Social mobility, health, and cardiovascular mortality

CAROLE HART, ${ }^{1}$ GEORGE DAVEY SMITH, ${ }^{2}$ DAVID BLANE, ${ }^{3}$ DAVID HOLE,${ }^{4}$ CHARLES GILLIS,${ }^{4}$ VICTOR HAWTHORNE ${ }^{1}$ ( Department of Public Health, University of Glasgow; ${ }^{2}$ Department of Social Medicine, University of Bristol; ${ }^{3}$ Academic Department of Psychiatry, Charing Cross and Westminster Medical School; ${ }^{4}$ West of Scotland Cancer Surveillance Unit.)

Objectives - To investigate variations in cardiovascular risk factors and cardiovascular mortality between social classes in terms of different social mobility experience. Design - Prospective cohort study.

Settings - Twenty seven workplaces in Glasgow, Clydebank, and Grangemouth.

Subjects - Nearly 6000 men of working age, screened between 1970 and 1973 .

Outcomes - Cardiovascular risk markers and cardiovascular mortality in a 21 year follow up period, analysed in terms of parental socia class, social class of first occupation and social class of occupation at time of screening.

Results - Social mobility between parental social class and current social class was clearly related to background factors. Height, years of education, and proportion with access to a car were highest in participants born in and remaining in non-manual social classes, next highest in those born in manual and changing to non-manual social classes, then in those born in non-manual and changing to manual social classes, and lowest in those born in and remaining in manual social classes. Similar results were seen in mean values of most cardiovascular risk factors. Higher $\mathrm{FEV}_{1}$, lower blood pressure, fewer current smokers and more former smokers were seen in participants born in and remaining in non-manual social classes. However, lower cholestero was seen in those born in and remaining in manual social classes. Similar findings with 
regard to risk factors were seen in relation to mobility between first and current social class. Cardiovascular mortality showed marked differences for manual compared with nonmanual social classes, whether indexed by parental, first, or current social class. Participants born in and remaining in non-manual social classes had significantly lower mortality rates than those born in and remaining in manual social classes, with those moving from manual to non-manual social classes having intermediate rates.

Conclusions - There were lower levels of cardiovascular risk factors and cardiovascular mortality in those experiencing upward social mobility. The worst outcomes were experienced by those remaining in manual social classes. Understanding this process could help reduce social differentials in mortality.

A prospective study of serum homocysteine concentration and risk of stroke in middle aged British men

I J PERRY, ${ }^{1} \mathrm{H}$ REFSUM, ${ }^{2} \mathrm{R}$ W MORRIS, ${ }^{1} \mathrm{~S}$ B EBRAHIM, ${ }^{1}$ P M UELAND, ${ }^{2}$ A G SHAPER ${ }^{1}\left({ }^{1} \mathrm{De}\right.$ partment of Public Health, Royal Free Hospital School of Medicine, London; ${ }^{2}$ Department of Pharmacology, University of Bergen, Norway) Background - Moderately raised levels of homocysteine in blood are common in the general population, reflecting inherited defects of enzymes modulating homocysteine metabolism and deficiency of nutritional cofactors, notably folic acid. There are consistent data from cross sectional and casecontrol studies linking moderate hyperhomocysteinaemia with cardiovascular disease, including ischaemic heart disease and stroke. However, we lack data from prospective, general population based studies.

Methods - We have examined the association between the serum homocysteine concentration and stroke in a nested case contro study within the British regional heart study cohort. Between 1978 and 1980, serum was saved from 5665 men, aged 40-59 years, randomly selected from one general practice population in each of 18 towns in Britain During follow up to December 1991 there were 141 incident cases of stroke among men without evidence of stroke at screening. Tota serum homocysteine was measured in 107 cases (with available serum stored at $-20^{\circ} \mathrm{C}$ ) and 118 controls. Controls were chosen from men who did not develop a stroke or myocardial infarction during follow up. Cases and controls were frequency matched by town and age group.

Results - Levels of homocysteine (geometric mean, $95 \% \mathrm{CI}$ ), were significantly higher in cases than controls: $13.7(12 \cdot 7,14.8) v 11.9$ $(11.3,12.6) \mu \mathrm{mol} / 1 ; \mathrm{p}=0.004$. There was a graded increase in the relative risk (odds ratio (OR)) of stroke in the 2nd, 3rd, and 4th quartile of homocystine (OR 1.3, (1.9, 2.9) trend $p=0.004$ ) relative to the first quartile. Adjustment for age, social class, body mass index, systolic blood pressure, cigarette smoking, forced expiratory volume, haematocrit, alcohol intake, and diabetes strengthened the associations between homocysteine concentration and risk of stroke, (adjusted 4th to 1 st quartile OR $5.9 ; 95 \%$ CI $1.8,18.9$ ). Among hypertensives, a homocysteine leve in the 4th quartile was associated with a higher risk of stroke, OR $3.7(95 \%$ CI 1.0 , 13.1) than among normotensives, OR 1.8
( $95 \%$ CI $0 \cdot 6,5 \cdot 5)$, though the difference was not significant on testing for interaction, $(p=$ $0 \cdot 2$ )

Conclusion - The data support the hypothesis of a causal link between blood homocysteine level and stroke. The possibility of an interaction with blood pressure merits examination in future studies. Trials of folate supplementation in the prevention of stroke are needed.

\section{Prevalence of Rose angina among women living in Britain}

M WARSKYJ, S FERRY, P HANNAFORD (Royal College of General Practitioners' Manchester Research Unit)

Introduction - The prevalence of angina among women living in Britain is not fully known, although a Scottish study of 5000 women aged $40-59$ years found that $8 \cdot 5 \%$ had Rose angina.

Objectives - To estimate the prevalence of Rose angina among women aged more than 40 years living throughout Britain, and to determine whether a number of factors influenced these rates.

Design - Since the late 1960s, the Royal College of General Practitioners' oral contraception study has observed an initial cohort of 47000 women in order to determine the effects of oral contraceptives. In November 1994, a self completed health survey questionnaire was sent to $1188(9.6 \%)$ of the 12356 women still under observation. The document included the Rose angina questionnaire and various lifestyle questions. $\mathrm{Re}$ minders were sent to non-responders in January and February 1995.

Results - A total of 992 questionnaires were returned and we were notified that anothe 21 women had died or moved away, giving corrected response rate of $85 \%$. Complete questionnaires were available for 813 women. Overall, 56 women had Rose angina $(6.9 \%$, $95 \%$ confidence interval (CI) $5 \cdot 2,8 \cdot 9 \%$ ); 45 grade I, 11 grade II. The prevalence rates varied with age $(<49$ years: $6.5 \%, 50-54$ years: $6 \cdot 9 \%, 55-59$ years: $8 \cdot 7 \%, 60-64$ years: $6 \cdot 0 \%, 65+$ years: $4 \cdot 8 \%$ ). Regional differences were apparent although there were few respondents in Wales Wales: $19.0 \%$, north England: $9 \cdot 4 \%$, south England: $5 \cdot 6 \%$, Scotland: $3 \cdot 4 \%$ ). Higher prevalence rates were reported by women in manual social classes $(7.6 \% v 5.7 \%$ in non-manual, difference $1.9 \%, 95 \%$ CI $-1 \cdot 6,5 \cdot 5 \%$ ), non-smokers $(7.3 \% v 6.6 \%$ in smokers, difference $0.7 \%$, $95 \% \mathrm{CI}-3.0,4 \cdot 2 \%$ ), ever users of oral contraceptives $(7 \cdot 2 \% v 6.4 \%$ in never users, difference $0 \cdot 8 \%, 95 \% \mathrm{CI}-2 \cdot 7,4 \cdot 3 \%$ ), ever users of hormone replacement therapy $(8.6 \%$ $v 6.3 \%$ in never users, difference $2.3 \%, 95 \%$ $\mathrm{CI}-1 \cdot 9,6 \cdot 6 \%$ ), and hysterectomised women $(12.3 \% \quad v \quad 5.5 \%$ in non-hysterectomised women, difference $6.8 \%, 95 \%$ CI 1.5 , $12 \cdot 2 \%)$. Angina was also more common among nulliparous women $(8 \cdot 3 \%)$ and those with three or more pregnancies $(9.6 \%)$, compared with those with one or two pregnancies $(5 \cdot 0 \%)$

Conclusions - Although based on relatively small numbers, these results suggest im portant variations in the prevalence of angina among women living in Britain. We are currently surveying the remaining 11168 women in the oral contraception study in order to increase the precision of the estimates.
CARDIOVASCULAR DISEASE MANAGEMENT (II)

Sex and socioeconomic differences in medical care for ischaemic heart disease

A NICHOLSON, H HEMINGWAY, K NANCHAHAL, M MARMOT (Department of Epidemiology and Public Health, University College London Medical School)

Aim - Given increased concern about equity in the treatment of ischaemic heart disease, sex and socioeconomic differences were examined for three stages of care: seeking advice, receiving a diagnosis, and receiving medication.

Design - Analysis of cross sectional data from the Whitehall II study using the Rose questionnaire for angina and the pain of possible myocardial infarction; ECG abnormality; and self reported doctor diagnosis and treatment. Employment grade was used as a measure of socioeconomic status.

Subjects - A total of 5766 men and 2589 women, aged 39 to 63 years.

Main outcome measures - Among those reporting pain on the Rose questionnaire or found to have an ECG abnormality, the outcomes of interest were: attending a doctor, receiving a diagnosis of ischaemia, and receiving drug treatment.

Results - Angina was more common in women $(5 \% \mathrm{~F}: 3 \% \mathrm{M}, \mathrm{p}<0.001)$ whereas a history of severe chest pain (lasting half an hour or more) ( $5 \% \mathrm{~F}: 6 \% \mathrm{M})$ and ECG abnormality $(4 \% \mathrm{~F}: 4 \% \mathrm{M})$ were approximately equal in the two sexes. (All percentages are age adjusted.) Nearly all men and women sought medical advice after experiencing severe chest pain $(92 \% \mathrm{M}: 86 \% \mathrm{~F})$ and there were no differences between grades. Men with symptoms or ECG abnormality were more likely than women to have a diagnosis of ischaemic heart disease $(23 \%$ of men with Rose angina and $12 \%$ of men with ECG abnormality had a diagnosis of ischaemia compared with $8 \%$ and $5 \%$ of women, $\mathrm{p}<0.001$ and $\mathrm{p}=0.02$ for sex differences). There was no evidence that grade of employment influenced diagnosis in men, but women with symptoms in the top employment grade were more likely to have had an ischaemic diagnosis than women in the lower grades. Among those diagnosed, men in higher grades were no more likely to report receiving medication.

Conclusion - Although there were no sex differences in seeking medical advice, women were less likely to receive a diagnosis of ischaemic heart disease. This may reflect differences in symptom perception. Socioeconomic status may influence diagnosis in women Although limited by the small numbers with a diagnosis, there is no evidence of social class differences in diagnosis or treatment in men.

Why do rates of coronary revascularisation vary geographically in the UK?

SUSAN LANGHAM, NICK BLACK, MARK PETTICREW (Health Services Research Unit, Department of Public Health and Policy, London School of Hygiene and Tropical Medicine)

Objective - To explain the reasons for geographical variation in the use of coronary revascularisation in the United Kingdom. 
Design - Cross sectional, ecological study. NHS and independent hospitals performing coronary revascularisation for the 11.6 million residents of South East Thames, East Anglian and North Western health regions in England plus the Greater Glasgow, Lanarkshire/Ayr and Arran health boards in Scotland.

Subjects - All residents aged 25 years or more in $1992 / 93$ who underwent coronary artery bypass grafting (CABG) or percutaneous transluminal angioplasty (PTCA) in either the public or private sector.

Main measures - Crude and age sex standardised intervention rates for residents of the 42 constituent districts and boards. Variation measured using the systematic component of variation.

Results - Considerable systematic variations in district rates of CABG and PTCA existed. These variations mostly arose from differences in supply factors. Higher rate districts were characterised by being close to a regional revascularisation centre and having a local cardiologist. Demand factors such as the level of need in the population (measured by coronary heart disease mortality) and the lack of use of alternative treatments not only failed to explain the observed variation but were inversely associated with the rate of intervention - an example of the inverse care law. The finding that the residents of more socially deprived districts experienced higher intervention rates was probably subject to confounding due to their close proximity to specialist centres.

Conclusions - If greater geographical equity of use for the same level of need is to be achieved then attention must be paid to the supply factors that determine levels of utilisation. As responsibility for purchasing these procedures is decentralised, utilisation might become even more unequal.

More equals better? The relationship between volume and outcome in coronary artery bypass graft surgery

A J SOWDEN, J J DEEKS, T A SHELDON, I S WATT (NHS Centre for Reviews and Dissemination, University of York)

Objective - To examine the evidence of a relationship between volume of coronary ar- tery bypass graft surgery and hospital mortality rates and assess the degree to which this could be due to bias.

Design - A literature review of empirical studies examining the relationship between volume and outcome of coronary artery bypass graft surgery. Data were extracted using the cut off closest to 200 procedures a year and patient numbers and adjusted mortality rates were extracted or calculated. Each study was given a score from 0 to 3 indicating the adequacy of adjustment for patient case mix. Logistic regression was used to model reported risk of death according to hospital volume.

Results - Fifteen studies were found but only six of these were included in the meta-analysis because of duplicate analyses of data sources and methods of reporting results. All studies reported a reduced mortality rate with increased volume of procedures (overall odds ratio $0.66 ; \mathrm{p}<0.001$ ). However, studies with better adjustment for confounding indicated less reduction in mortality with volume (odds ratio of adjustment-volume interaction $1 \cdot 15$; $\mathrm{p}=0.04$ ).

Conclusions - The evidence for reduced mortality in hospitals with high volume of coronary artery bypass graft surgery is based entirely on observational studies. Such studies are susceptible to biased estimates of benefit because of inadequate adjustment for differences in case mix between hospitals. This study suggests that previous studies may have overestimated the benefit of increased volume, and signals the need for caution in interpreting the results of observational studies of volume and patient outcomes.

Death after acute myocardial infarction: how many league tables do you need?

alastair h Leyland (Public Health Research Unit, University of Glasgow)

Objective - To investigate how hospital outcomes vary according to the definition of the outcome and the inclusion of non-hospital deaths.
Subjects - Hospital discharge records from 21834 patient episodes in Scotland during the period December 1992 to November 1993 relating to episodes in which the principal diagnosis was acute myocardial infarction (AMI, ICD 410) and their death records, if appropriate, together with 9146 death records from patients whose cause of death was AMI and who had not been admitted to hospital during the study period. The hospital discharge records are drawn from the routine system of Scottish morbidity records (form SMR1)

Main outcome measure - Mortality (taken from death records obtained from the Registrar General for Scotland).

Methods - Multilevel modelling of patient records within the hierarchies of postcode sector, local government district, and hospital. Results - The 21834 patient episodes during this year were generated by 13819 patients. The 30 day mortality rates after admission to hospital range from $15 \%$ to $28 \%$ among the larger hospitals on a per-patient basis and from $12 \%$ to $27 \%$ on a per-episode basis; however, the ranking of hospitals is not the same for the two measures. Similarly, the relative positions are affected if the follow up period is changed from 30 days or if the period of follow up is taken as commencing on the date of admission or the date of discharge. All of the above change again if the outcomes are standardised for age, sex, and comorbidities. One problem of the use of mortality after hospital admissions is that $40 \%$ of cases of AMI died before reaching hospital such figures are typically excluded from the analysis of hospitals, although they provide information about localities and the patients' access to hospital. There is considerable disparity between small areas in the proportion of patients who died before reaching hospital. Conclusions - The sensitivity of the results, when expressed in any kind of "league table", to the precise definition of the outcome suggests that any rankings are unstable and raises questions as to whether there can ever be a single, definitive measure of hospital performance. The inclusion of non-hospital mortality is as important as standardisation in terms of adjusting for factors not in the control of the hospitals themselves. 
of health, such tools do not attain the degree of rigour demanded of a precise scientific instrument. More worryingly, the text could also be criticised from the very perspective that it intends to promote. It could be argued that a fundamental tension exists between the relatively enlightened thoughts on concepts of health contained within the introductory chapter and the highly formal and quantified tools that subsequently follow. In reviewing discrepancies between the SF-36 measure of general health and associated qualitative assessments of health, Hill, Harries, and Popay ${ }^{1}$ have already suggested that irrespective of any intended sensitivity, quantitative tools can never match the subtlety gained from more open ended methods. This tension is left largely unresolved in the text.

Bowling herself points out that it is easy to criticise established tools. It is also arguable that in a policy context which demands quantitative data to inform decision making, such tools are practical, pragmatic, and inherently useful. If this is the case, Bowling's book provides an important step towards the development of measures of quality of life that are both sensitive and rigorous.

SANDY WHITELAW Lecturer in Health Promotion University of Manchester

1 Hill S, Harries U, Popay J. Patients' assessment of the outcomes of their care. PHRRC report 3 July 1994

\section{BOOKS RECEIVED}

APHA. Chronic Disease Epidemiology and Control. ( $\mathrm{Pp} 358 ; £ 22.50)$ APHA, 1993. ISBN: 0-87553-214-4.

B K Armstrong, E White, R Caracci, Principles of Exposure, Measurement in Epidemiology, (Pp 351; 25.00$)$. OUP, 1994. ISBN: 0-19-262020-7.

A Bowling. Measuring Disease. (Pp 274; $£ 37.50$ hbk, $£ 16.99$ pbk). Buckingham: Open University Press, 1995. ISBN: 0335-19225-4.

P A Braveman, E Tarimo. Screening in Primary Health Care. (Pp 196; \$27.10). New York: WHO, 1994. ISBN: 924-154473-2.

D Coggon. Statistics in Clinical Practice. (Pp 116; 1 10.95). London: BMJ Books, 1995. ISBN: 0-7279-0907 X.

M C Colquhoun, A J Handley, T R Evans (eds). ABC of Resuscitation (3rd ed) (Pp 88; f12.95). London: BMJ Books, 1995. ISBN: 0-7279-0839-1.

B Davey, A Gray, C Seale (eds). Health and Disease: A Reader. Pp 436; $£ 40.00$ hbk, $£ 14.99$ pbk). Buckingham: Open University Press, 1995. ISBN: 0-335-19209-2.

P S W Davies, T J Cole (eds). Body Composition Techniques in Health and Disease. ( $\mathrm{Pp} 282 ; £ 37.50)$. Cambridge: Cambridge University Press, 1995. ISBN 0521-46179-0.

J M Dixon (ed). ABC of Breast Diseases.
(Pp 74; £12.95). London: BMJ Books, 1995. ISBN: 0-7279-0915-0.

G Edwards. Alcohol and the Public Good. (Pp 226; £14.95). Oxford: Oxford Medical Publications, 1994. ISBN 0-19-262561-6.

M A Favia. Vandals at the Gates of Medicine. (Pp 403; \$41.95) Hacienda Publications, 1994.

$P$ Foster. Women and the Health Industry. (Pp 218; $\{37.50 \mathrm{hbk}, £ 12.99$ pbk). Oxford: Oxford University Press, 1995. ISBN: 0335-09472-4.

J Fry, J Horder. Primary Health Care in an International Context. London: Nuffield Provincial Hospitals Trust, 1994. ISBN: 0900574-90-9.

J Garcia, M France-Dawson, A Macfarlane. Improving Infant Health. ( $£ 5.99)$. London: Health Education Authority, 1994 ISBN: 0-7521-0230-3.

J Gomez. Living with Diabetes. (Pp 133; $£ 6.99$ ). London: Sheldon Press, 1995. ISBN: 0-85969-723-1.

A Greese, D Parker. Cost Analysis in Primary Health Care. (Pp 147; SwFr 25). Geneva: WHO, 1994. ISBN: 92-4-154470-8.

T Harpham, I Blue (eds), Urbanization and Mental Health in Developing Countries. (Pp 266; $£ 32.50$ ). Aldershot: Avebury, 1995 ISBN: 1-85628-971-0.

Health Education Authority. Alcohol: Intervening in Primary Care. ( $£ 4.99)$. Lon don: HEA, 1994. ISBN: 0-7521-0229 X.

O Hockwin, K Sasaki (eds). Developments in Ophthalmology. (Pp 100; \$94.50). Basel: Karger Press, 1993. ISBN: 3-8055-5987-9.

J Hunt. Coping Successfully with Your Irritable Bladder. (Pp 82; $£ 5.99$ ). London: Sheldon Press, 1995. ISBN: 0-86969-717-7.

$C$ Jenkinson, Measuring Health and Medical Outcomes. (£35.00). London: UCI Press. ISBN: 1857280830 (hbk); $1-$ 85728-084-9 (pbk).

P Johnstone, J Ranken, Management Support for Primary Health Care. $(£ 12.00)$ FSG Communications, 1994. ISBN: $1-$ 871180-20-4.

King's Fund. Tackling Inequalities in Health. (Pp 166). London: King's Fund 1995. ISBN 1-85717-088-1.

J M Last (ed). A Dictionary of Epidemiology (3rd ed) (Pp 180). Oxford: Oxford University Press, 1995. ISBN: 019-509668-1.

Laukinen, Bergstrom, Makela, Petomaa, Health and Disease in Developing Countries. (Pp 586). London: Macmillan, 1994 ISBN: 0-333-589000-9.

J Corbett McDonald (ed). Epidemiology of Work Related Diseases. (Pp 498; $£$ 49.95) London: BMJ Books, 1995. ISBN: 07279-0856-1

M Marinker (ed). Medical Audit and General Practice (2nd ed) (Pp 224). London: BMJ Books, 1995. ISBN: 0-7279-0908-8.

C G N Mascie-Taylor, B Bogin, Human variability and plasticity. (Pp 241, 35.00). Cambridge: Cambridge University Press, 1995. ISBN: 0-521-45399-2.

P Meers, J Sedgwick, M Worsley, Microbiology and Epidemiology of Infection for Health Science Students. (Pp 330 14.99). London: Chapman and Hall, 1995. ISBN: 0-412-59900-7.

Mielck, Giraldes, Health Inequalities. (Pp 235). Waxmann, 1995. ISBN 389325-299-1.
C S Muir, P McKinney. A Strategy for Cancer Control in Scotland. Glasgow: Scottish Forum for Public Health Medicine. ISBN: 0-95187-422-5.

National Research Council. Radiation Dose Reconstruction for Epidemiologic Uses. (Pp 138; $\{24.95)$. Oxford: National Academy Press, 1995. ISBN: 0-309-05099-5.

E T O'Brien, D G Beevers, H J Marshall, ABC of Hypertension (3rd ed) (Pp 79 $£ 12.95)$. London: BMJ Books, 1995. ISBN: 0-7279-0769-7.

D M Oleske (ed.) Epidemiology and the Delivery of Health Care Services: Methods and Applications. (Pp 235; $\$ 45.00)$. New York: Plenum Publishing, 1995. ISBN: 0-306-44968-4.

A F Olshan, D Mattison (ed). Male-mediated Developmental Toxicity. (Pp 406; $\$ 115.00)$. New York: Plenum Publishing, 1994. ISBN: 0-306-44815-7.

OPCS. OPCS Longitudinal Study 19711991. (Pp 374; $£ 27.30$ ). London: HMSO, 1995. ISBN: 0-11-691637-0.

NOTICES

International Congress on Women, Work and Health. Barcelona, 18/20 April 1996. For further information, please contact: Dona, Salut i Qualitat de Vida, C/Paris 150 $1^{0} 2^{\mathrm{a}}$, Barcelona 08036, Spain. Tel/fax: 322 6554 .

16th International Congress of Nutrition. Montreal, QC, Canada, 27 July-1 August 1997. For further information, please contact: Congress Secretariat, IUNS '97, National Research Council Canada, Building M-19, Montreal Road, Ottawa, ON, Canada K1A 0R6. Tel: (613) 993-7271. Fax: (613) 9937250

16th Annual New England Epidemiology Summer Program. 10 June-5 July 1996, 5 and 10 day courses at Tufts University in Medford/Boston, Massachusetts. For more information contact: The New England Epidemiology Institute, Dept PA-JECH, One Newton Executive Park, Newton Lower Falls, MA 02162-1450. Tel: (617) 244-9669; e-mail: epidemiol@aol.com.

\section{Corrigendum}

Smoking and health promotion in Nazi Germany

The letter which appeared in October 1995 (49(5):555) was submitted by George Davey Smith and Matthias Egger. The letter is reprinted on $\mathrm{p} 109$.

Society for Social Medicine Abstracts The abstracts which appeared in the October 1995 issue (vol 49(5):534-54) were from the September 1995 scientific meeting of the Society for Social Medicine, not the 1994 meeting. 